This is a post-peer-review, pre-copyedit version of an article published in Environmental Monitoring and Assessment. The final authenticated version is available online at: http://dx.doi.org/10.1007/s10661-020-8085-9.

(C) 2019 Springer Nature Switzerland AG. 


\title{
Evaluation of Salix alba, Juglans regia and Populus nigra as biomonitors of PTEs in the riparian soils of the Sava River
}

Zorana Mataruga $^{1}$, Snežana Jarić ${ }^{1}$, Milica Marković ${ }^{1}$, Marija Pavlović ${ }^{1}$, Dragana Pavlović ${ }^{1}$, Ksenija Jakovljević Miroslava Mitrović ${ }^{1}$, Pavle Pavlović ${ }^{1}$

${ }^{1}$ Department of Ecology, Institute for Biological Research 'Siniša Stanković', University of Belgrade, Bulevar despota Stefana 142, Belgrade, Serbia

${ }^{2}$ Faculty of Biology, Institute of Botany and Botanical Garden 'Jevremovac', University of Belgrade, Takovska 43, 11000 Belgrade, Serbia

\section{Corresponding author:}

Zorana Mataruga,

E-mail: zorana.mataruga@ibiss.bg.ac.rs

ORCID: https://orcid.org/0000-0003-3353-4810

\begin{abstract}
A large number of human activities result in the release of potentially toxic elements (PTEs) into the environment, which could lead to the degradation of riparian areas. This study aimed to evaluate the potential of Salix alba, Juglans regia and Populus nigra for the biomonitoring of PTEs in the riparian soils of the River Sava. Levels of seven PTEs (As, Cd, $\mathrm{Cr}, \mathrm{Cu}, \mathrm{Ni}, \mathrm{Pb}$ and $\mathrm{Zn}$ ) were measured in the soils, roots and leaves of plants at selected sampling sites and evaluated according to bioaccumulation and translocation factors. The obtained results showed that in riparian soils $\mathrm{As}, \mathrm{Cr}, \mathrm{Cu}, \mathrm{Ni}$ and $\mathrm{Zn}$ were at levels considered to be critical for plants. The levels of As, Cd, Cr, Ni and Zn measured in roots of Salix alba and As, Cr, Ni and $\mathrm{Zn}$ in its leaves were toxic for plant tissue. Toxic levels of $\mathrm{Cr}$ were also measured in the roots of Juglans regia and As in its leaves, as well as As and $\mathrm{Cr}$ in the roots of Populus nigra, and $\mathrm{Zn}$ in its leaves. Bioconcentration and translocation factors showed that S. alba and P. nigra have potential for the phytoextraction of $\mathrm{Zn}$ and $\mathrm{Cd}$, while J. regia has potential for the phytoextraction of As. In terms of phytostabilisation potential, S. alba proved to be good for the phytostabilisation of $\mathrm{Cd}$ and $\mathrm{Cu}$, and J. regia for the phytostabilisation of $\mathrm{Cr}$, As, $\mathrm{Ni}$ and $\mathrm{Pb}$, while $P$. nigra showed potential for the phytostabilisation of $\mathrm{Cr}, \mathrm{Ni}, \mathrm{Pb}$ and $\mathrm{Cu}$.
\end{abstract}

Keywords: Salix alba, Juglans regia, Populus nigra, riparian zone, potentially toxic elements, spatial variations 


\section{Introduction}

Riparian areas are transitional zones between terrestrial and aquatic ecosystems which provide specific conditions for diverse life forms (Oelbermann and Raimbault 2015). They are habitats for a large number of aquatic and terrestrial species and can affect how they disperse, meaning they are important for biodiversity conservation (Pennington et al. 2010; Sabater and Elosegi 2013). Besides the benefits that riparian areas offer for wildlife, they also provide a large number of ecosystem services for humans, such as food, transportation, recreation and tourism, as well as power production and waste disposal (Naiman and Decamps 1997; Pennington et al. 2010). However, the rapid development of industrialisation, urbanisation, and agricultural practices has led to the degradation of riparian areas and a loss of riparian vegetation, which in turn has caused a deterioration in freshwater ecosystems (Sunil et al. 2010).

The ecological importance of the River Sava and its riparian zone can be seen in the significant number of protected areas. The River Sava is the third longest tributary of the River Danube and it extends across four countries (Slovenia, Croatia, Bosnia and Herzegovina, and Serbia) with a catchment area of 97,713 $\mathrm{km}^{2}$. Around $36 \%$ of the floodplain and 64\% of the River Sava's water body are protected by law (Schwarz 2016). There are 167 protected areas in total, including 6 Ramsar Sites and 8 national parks, as well as numerous important bird and plant areas, areas protected at the national level and Natura 2000 sites (ISRBC, 2009). Many human activities result in the release of various pollutants in the riparian zone of large rivers; of these, particular attention is paid to potentially toxic elements (PTEs) and the impact they have on the environment. Numerous studies have reported that riparian habitats have a large capacity to accumulate PTEs (Punshon et al. 2003; Du Laing et al. 2009; Milačič et al. 2017; Pavlović et al. 2019), with As, Cd, Cr, Cu, Ni, Pb and Zn having a damaging effect on living organisms, including plants (Gjorgieva et al. 2011; Zhang et al. 2011; Pavlović et al. 2016). Mainly originating from industrial and municipal waste waters and pollutants from agricultural activities, pollution of the Sava River with PTEs exhibits a spatial trend: in the upper reaches mineral weathering predominates, in the middle reaches there is pollution arising from agricultural activities, while high levels of pollutants originate from industrial processing, including untreated municipal wastewater discharge, in the lower reaches (Ščančar et al. 2015).

In order to quantify the quality of the environment, in terms of pollution from PTEs, a large number of higher plants are used as biomonitors (Piczak et al. 2003; Madejon et al. 2013). Trees are not the best indicators of environmental quality, but they have a wide distribution and are long living, so they can be used in the biomonitoring of large areas (Sawidis et al. 2011). The other advantages of using trees as biomonitors are the simplicity of sampling and identification, the ample supply of biological material for analyses, and the high biomass for PTE accumulation (Berlizov et al. 2007; Sawidis et al. 2011). Native species are more resistant than those introduced from other regions, bearing in mind their higher levels of survival, growth and reproduction success (Shu et al. 2002; Yoon et al. 2006). Therefore, there is continual interest in finding native species that are tolerant to increased PTE levels.

Previous research has shown that Salix and Populus species generally possess a high tolerance to PTE pollution, which is why they are proposed for the biomonitoring, stabilisation and removal of PTEs from 
contaminated soils (Mertens et al. 2004; Laureysens et al. 2005; Wu et al. 2010; Gaudet et al. 2011; Bhargava et al. 2012; Chen et al. 2014; Pavlović et al. 2016). Up to now, Salix alba L. and Populus nigra L. have not been evaluated as biomonitors of PTEs in the Sava River Basin. Both species are fast growing, easily propagated and tolerant to diverse types of soils (Kuzovkina et al. 2004). In addition to the dominant species of the Salix and Populus genera, another abundant species on the alluvial, well-drained soils of the River Sava is Juglans regia L. (Jovanović 1970). In addition to growing spontaneously in nature, this species is often cultivated, too. However, a large number of studies focus on PTE levels in the shells or kernels of J. regia (Arpadjan et al. 2013; Tošić et al. 2014; Feizi and Jalali 2015), while there is lack of data to confirm whether this species is suitable as a biomonitor of PTEs.

In this respect, the main objectives of this research were to evaluate the potential of native, naturally growing species in the River Sava riparian zone - Salix alba, Juglans regia and Populus nigra - for the uptake, accumulation and translocation of PTEs. These included: 1) determining PTE content in riparian soils; 2) determining soil enrichment with PTEs; 3) determining PTE content in the roots and leaves of S. alba, J. regia and P. nigra; 4) evaluating PTE uptake, accumulation and translocation through bioaccumulation and translocation factors; 5) highlighting variations between and discovering patterns of PTE accumulation in the analysed species; and 6) investigating the potential use of species as biomonitors of PTEs.

\section{Materials and methods}

Study area

This study was conducted along the River Sava, at 12 selected localities in Slovenia (Mojstrana (MOJ), Radovljica (RAD), Litija (LIT), Vrhovo (VRH), Catez (CAT)), Croatia (Zagreb (ZAG), Jasenovac (JAS), Slavonski Brod (SLB), Zupanja (ZUP)), and Serbia (Sremska Mitrovica (SRM), Sabac (SAB), Belgrade (BEO)) (Table 1; Fig. 1). The main characteristics of the Sava River Basin (SRB) have already been discussed extensively (Dragun et al. 2015; Ogrinc et al. 2015; Ščančar et al. 2015).

The SRB has a diverse geological composition, with magmatic (granite, diabase, dacite, andesite and peridotites), metamorphic (schist, gneiss, marble and quartzite) and sedimentary rocks (limestone, dolomites and conglomerates), and limestone as the dominant geological substrate (Simić et al. 2015; ISRBC 2016; Schwarz 2016). Soils in the upper section of the river are undeveloped skeleton soils, accompanied by poorly developed brown floodplain soils. In lowland parts, gley and semi-gley fluvisols are most dominant (Schwarz 2016). In terms of land use, forests and semi-natural areas cover $54.7 \%$ of the land, $42.4 \%$ is used for agricultural activities, while artificial surfaces (roads, buildings, etc.) cover 2.2\% (ISRBC 2009).

The climate of the SRB is moderate continental, with the exception of the higher altitudes, where an alpine (mountainous) climate prevails. Average annual precipitation and air temperature differ along the course of the river. The annual mean air temperature in the upper stretches of the river is $6{ }^{\circ} \mathrm{C}$, while in the lower stretches (near the confluence with the Danube) it reaches $13^{\circ} \mathrm{C}$. Annual precipitation varies from $660 \mathrm{~mm}$ year

${ }^{1}$ at the mouth of the River Sava up to 2000 to $3000 \mathrm{~mm}$ year $^{-1}$ in the alpine region. Maximum flows are usually 
recorded in spring and low ones in autumn, which is in line with precipitation and snow melting patterns (Ogrinc et al. 2015).

The floristic differentiation of riparian vegetation within the SRB is mainly affected by different hydrological conditions, such as intensity and duration of flooding and groundwater level, as well as topography and soil properties (maximum water capacity, aeration and water permeability). The woody vegetation consists of riparian forests of different willow species and white and black poplars (Populus alba and P. nigra) (Schwarz 2016). Besides the dominant species of the genera Salix and Populus, Alnus glutinosa (L.) Gaertn., Fraxinus angustifolia Vahl, Ulmus laevis Pall., Ulmus glabra Huds., Quercus robur L. and Juglans regia L. are frequent species in these forests (Karadžić et al. 2015). Willow communities along the River Sava belong to the alliances Salicion eleagno-daphnoidis (Moor 1958) Grass 1993 and Salicion albae Soó 1930 of the class Salicetea purpureae Moor 1958. The alliance Salicion albae grows on soils with a greater water capacity, mainly gravel and sandy littoral; it is directly impacted by streams and experiences frequent flooding, being just above the mean water level. White willow communities are dominated by S. alba, which is sometimes accompanied by $S$. purpurea and S. triandra (Karadžić et al. 2015). Forests of P. nigra are rare, but in mixed communities with willow (Salici albae-Populetum nigrae (R. Tx. 1931) Meyer Drees 1936) and white poplar (Populetum nigraealbae Slavnic 1952), P. nigra is the dominant species of riparian forests. Juglans regia is found in a large number of localities in the study area as an accompanying species in willow and poplar forests.

Sampling

Sample collection was conducted in September 2015 during the GLOBAQUA expedition. Sampling sites were selected based on sample accessibility, as well as site representativeness in terms of sources of pollution (e.g. agricultural and urban activities, industry, traffic, etc.) (Fig 1; Table 1). Soil samples were collected along the river bank, with five subsamples taken at each sampling site, from a depth of $0-10 \mathrm{~cm}$. At each sampling site, these five subsamples were mixed in order to form a representative composite sample. The composite samples were collected in PVC buckets and stored at $4{ }^{\circ} \mathrm{C}$, in the dark. Soil samples were air-dried and sieved through a 0.2 mm stainless sieve for chemical analyses.

Plant samples were collected from three to five individuals of S. alba, J. regia and P. nigra trees of approximately the same age by random selection. Approximately $30 \mathrm{~g}$ of mature leaves were collected from each tree at a height of 1.5 - $2 \mathrm{~m}$ above the ground, from all sides of the tree. A composite plant (leaves and roots) sample for each examined species was formed by mixing (three to five) subsamples at each sampling site. Salix alba was found at all 12 sampling sites, forming a total of 12 composite samples. Populus nigra was found at the following localities: MOJ, VRH, CAT, ZAG, JAS, ZUP, SRM, SAB and BEO, forming a total of 9 composite samples, while J. regia was found at MOJ, RAD, LIT, VRH, CAT, JAS and SRM, forming a total of 7 composite samples (Fig. 1). Root samples were taken from the same tree individuals in the zone of the rhizosphere. Leaf and root samples were washed with tap and deionised water in order to remove all soil residues from their surfaces and then dried to a constant weight at $75{ }^{\circ} \mathrm{C}$ (Binder, Tuttlingen, Germany). Samples were sieved with a laboratory mill (Polymix, Kinematica AG) through a $1.5 \mathrm{~mm}$ stainless steel sieve before being analyzed for PTE content. 


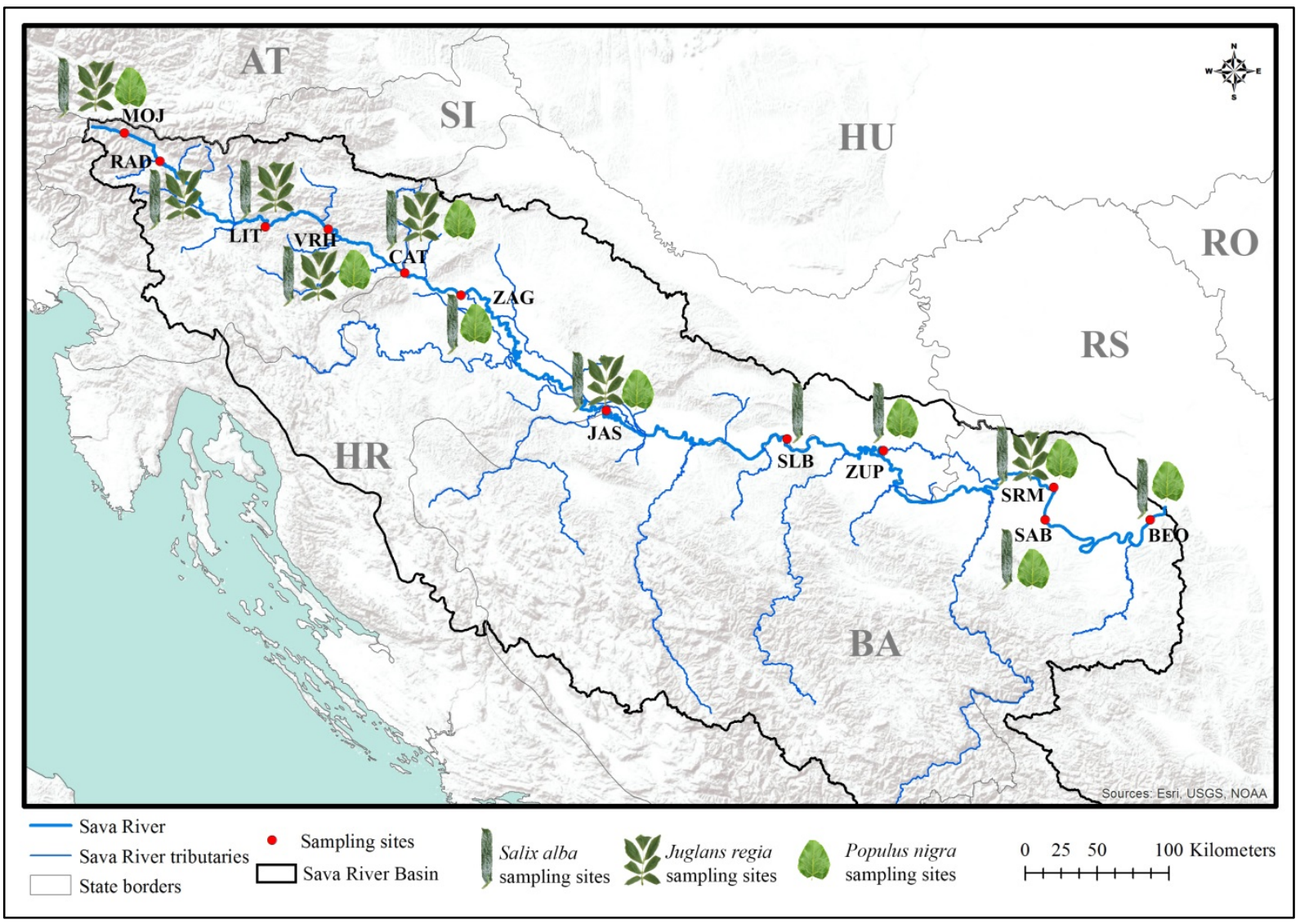

Fig. 1 Sampling sites along the Sava River

Table 1 Description of sampling sites

\begin{tabular}{|c|c|c|c|c|c|c|c|}
\hline Abbreviation & Full name & State & $\begin{array}{l}\text { Latitude } 1 \\
\quad\left({ }^{\circ}\right)\end{array}$ & $\begin{array}{l}\text { Longitude } \\
\left.\quad{ }^{\circ}\right)\end{array}$ & $\begin{array}{l}\text { Elevation } \\
\text { (m asl) }\end{array}$ & $\begin{array}{l}\text { Distance } \\
\text { from } \\
\text { the } \\
\text { mouth } \\
\text { (rkm) } \\
\end{array}$ & Pollution sources \\
\hline MOJ & Mojstrana & Slovenia & 46.459967 & 13.940096 & 661 & 930 & $\begin{array}{l}\text { No direct sources of pollution; } \\
\text { Elevated content of PTEs is } \\
\text { from parent rock weathering }\end{array}$ \\
\hline RAD & Radovljica & Slovenia & 46.339529 & 14.163860 & 409 & 908 & $\begin{array}{l}\text { Metal industry is located } \\
\text { upstream of the sampling site }\end{array}$ \\
\hline LIT & Litija & Slovenia & 46.066067 & 14.850483 & 225 & 810 & $\begin{array}{l}\text { Abandoned mining and } \\
\text { agricultural activities }\end{array}$ \\
\hline VRH & Vrhovo & Slovenia & 46.042900 & 15.226300 & 194 & 776 & $\begin{array}{l}\text { Dam, hydromorphological } \\
\text { change to river flow }\end{array}$ \\
\hline CAT & Catez & Slovenia & 45.890362 & 15.630107 & 137 & 736 & $\begin{array}{l}\text { Viticulture and urban } \\
\text { activities, wood processing } \\
\text { industry }\end{array}$ \\
\hline ZAG & Zagreb & Croatia & 45.785695 & 15.981591 & 112 & 664 & Industrial and urban activities \\
\hline JAS & Jasenovac & Croatia & 45.263670 & 16.894265 & 90 & 489 & $\begin{array}{l}\text { River traffic and agricultural } \\
\text { activities }\end{array}$ \\
\hline SLB & $\begin{array}{l}\text { Slavonski } \\
\text { Brod }\end{array}$ & Croatia & 45.144906 & 17.984106 & 82 & 360 & $\begin{array}{l}\text { Oil industry, river traffic and } \\
\text { agricultural activities }\end{array}$ \\
\hline
\end{tabular}




\begin{tabular}{cccccccl} 
ZUP & Zupanja & Croatia & 45.075484 & 18.686883 & 77 & 262 & $\begin{array}{l}\text { Oil, metal and mining } \\
\text { industry, river traffic and } \\
\text { agricultural activities }\end{array}$ \\
SRM & $\begin{array}{l}\text { Sremska } \\
\text { Mitrovica }\end{array}$ & Serbia & 44.91357519 .752491 & 72 & 118 & $\begin{array}{l}\text { Heavy industry, urban and } \\
\text { agricultural activities, river } \\
\text { traffic }\end{array}$ \\
SAB & Sabac & Serbia & 44.76990019 .699400 & 71 & 106 & $\begin{array}{l}\text { Chemical industry, urban and } \\
\text { agricultural activities, river } \\
\text { traffic }\end{array}$ \\
BEO & Belgrade & Serbia & 44.76851120 .355560 & 69 & 14 & & $\begin{array}{l}\text { Untreated municipal water, } \\
\text { thermal power plant, industrial } \\
\text { and urban activities, river } \\
\text { traffic }\end{array}$ \\
\hline
\end{tabular}

Soil and plant analysis

Soil sample mineralisation was carried out by wet digestion in a microwave (CEM Mars 6) using an aqua regia mixture (3 ml of $65 \% \mathrm{HNO}_{3}$ and $9 \mathrm{ml}$ of $37 \% \mathrm{HCl}$ ), while the content of PTEs (As, $\mathrm{Cd}, \mathrm{Cr}, \mathrm{Cu}, \mathrm{Ni}, \mathrm{Pb}$ and $\mathrm{Zn}$ ) was determined by inductively coupled plasma optic spectrometry (ICP-OES, Spectro Genesis). The accuracy of the results dwas confirmed by analysing standard reference soil material (Loam soil - ERM-CC141, IRMM certified by EC-JRC). The recovery values found were within 90 - 110\%.

Plant sample mineralisation was also carried out by wet digestion in a microwave (CEM Mars 6) using a mixture of $9 \mathrm{ml}$ of $65 \% \mathrm{HNO} 3$ and $3 \mathrm{ml}$ of 30\% H2O2. The accuracy of the measured results was confirmed by analysing the standard reference material (Beach leaves - BCR-100, IRMM certified by EC-JRC). The recovery values found were within 91.7 - 108.9\%

All samples were analyzed in 6 replicates and results were presented as mean values, with standard deviation (SD). The detection limits for the analysed elements in soil and plant samples were as follows (mg $\mathrm{kg}^{-1}$ ): As $-0.005, \mathrm{Cd}-0.0002, \mathrm{Cr}-0.001, \mathrm{Cu}-0.001, \mathrm{Ni}-0.0003, \mathrm{~Pb}-0.004$, and $\mathrm{Zn}-0.006$.

Data analysis

The enrichment factor (EF) has been widely used to assess the environmental burden of elements accumulated in soils (Hu et al. 2013; Čakmak et al. 2018), as well as to evaluate possible anthropogenic inputs of PTEs in soil (Swarzenski et al. 2006; Bai et al. 2015). The EF was calculated as:

$E F=\frac{\left(\frac{C_{x}}{C_{M n}}\right) \text { soil }}{\left(\frac{C_{x}}{C_{M n}}\right) \text { background }}$

where $\left(C_{x} / C_{M n}\right)$ soil is the ratio between concentrations of the potentially enriched element $C_{x}$ and $M n$ concentrations $\left(\mathrm{C}_{\mathrm{Mn}}\right)$ in the same soil sample, and $\left(\mathrm{C}_{\mathrm{x}} / \mathrm{C}_{\mathrm{Mn}}\right)$ background is the ratio of the referenced background values. The use of Mn as the reference background element has been referred to in studies by Barbieri (2016), Kowalska et al. (2018), and Pavlović et al. (2019). To calculate the EF, the background values published in Marković et al. (2018) were used. According to Sutherland (2000), five contamination categories are recognised 
based on the enrichment factor: $\mathrm{EF}<2$ represents deficiency to minimal enrichment; EF $2-5$, moderate enrichment; EF 5 - 20, significant enrichment; EF 20 - 40, very high enrichment; and EF > 40, extremely high enrichment. In addition, an EF value of around 1 could indicate the predominant natural origin of the element in soil (Mil-Homens et al. 2006).

In order to evaluate (potential) toxic element tolerance in selected plant species, the bioaccumulation (BCF) and translocation (TF) factors were calculated as follows:

$B C F=\frac{C_{\text {root }}}{C_{\text {soil }}}$

where $\mathrm{C}_{\text {root }}$ represents the content of the selected element in roots and $\mathrm{C}_{\text {soil }}$ represents the content of the same element in soil (Zayed et al. 1998), and

$T F=\frac{C_{\text {leaf }}}{C_{\text {root }}}$

where $\mathrm{C}_{\text {leaf }}$ represents the content of the selected element in leaves and $\mathrm{C}_{\text {root }}$ represents the content of the same element in root samples (Li et al. 2007; Malik et al. 2010).

In order to determine the differences in the content of the chemical elements obtained in soil and plant material, a one-way ANOVA was carried out, with Tukey's honest significant difference (HSD) post-hoc test. ANOVA was conducted for each examined species separately. Correlations between the levels of the examined elements in soil, root and leave samples were obtained using non-parametric Spearman rank-order correlation. Canonical discriminant analysis (CDA) was performed for element contents in roots and leaves separately in order to classify species according to different accumulation abilities. Descriptive and multivariate statistical analyses were performed using Statistica 10.0 software.

\section{Results and discussion}

PTEs in soil

The element levels established in soil are presented in Table 2. The trend for the average element content for the entire study area was as follows: $\mathrm{Zn}>\mathrm{Cr}>\mathrm{Cu}>\mathrm{Ni}>\mathrm{Pb}>\mathrm{As}>\mathrm{Cd}$. Compared to the average element content in world soils (Kabata-Pendias 2011), a higher element content was measured for: As (> $8.4 \mathrm{mg} \mathrm{kg}^{-1}$ ) at the ZUP, SRM, SAB and BEO sampling sites, Cd (>0.45 mg kg-1) at BEO, Cr (> $\left.51 \mathrm{mg} \mathrm{kg}^{-1}\right)$ at ZUP, SRM, SAB and BEO, Cu (> $\left.23 \mathrm{mg} \mathrm{kg}^{-1}\right)$ at CAT, ZUP, SRM, SAB and BEO, Ni (> $\left.26 \mathrm{mg} \mathrm{kg}^{-1}\right)$ at SLB, ZUP, SRM, SAB and BEO, Pb (> $\left.28 \mathrm{mg} \mathrm{kg}^{-1}\right)$ at MOJ, SRM, SAB and BEO, and $\left.\mathrm{Zn} \mathrm{(>60} \mathrm{mg} \mathrm{kg}^{-1}\right)$ at MOJ, VRH, CAT, ZUP, SRM, SAB and BEO. The background values for European soils (Gawlik and Bidoglio 2006) were exceeded for: $\mathrm{Cr}$ (> $\left.100 \mathrm{mg} \mathrm{kg}^{-1}\right)$ at ZUP, SAB and BEO, Cu (> $\left.140 \mathrm{mg} \mathrm{kg}^{-1}\right)$ at CAT, and $\mathrm{Ni}\left(>75 \mathrm{mg} \mathrm{kg}^{-1}\right)$ at ZUP, SRM, SAB and BEO. Compared to the background values for this area recorded in the study by Marković et al. (2018), a higher content was measured for: As (> $\left.11.53 \mathrm{mg} \mathrm{kg}^{-1}\right)$ at SRM, SAB and BEO, Cd (>0.68 $\left.\mathrm{mg} \mathrm{kg}^{-1}\right)$ at BEO, Cr $\left(>72.52 \mathrm{mg} \mathrm{kg}^{-1}\right)$ at ZUP, SRM, SAB and BEO, Cu (> $\left.24.12 \mathrm{mg} \mathrm{kg}^{-1}\right)$ at CAT, ZUP, SRM, SAB and BEO, Ni $\left(>41.33 \mathrm{mg} \mathrm{kg}^{-1}\right)$ at ZUP, SRM, SAB and BEO, Pb (> $\left.44.03 \mathrm{mg} \mathrm{kg}^{-1}\right)$ at MOJ, SAB and BEO, and Zn (> 91.64 $\mathrm{mg} \mathrm{kg}^{-1}$ ) at MOJ, CAT, SRM, SAB and BEO. Levels in the critical range for plants (Alloway 2013) were 
measured for: As (20 - $50 \mathrm{mg} \mathrm{kg}^{-1}$ ) and $\mathrm{Cr}\left(75-100 \mathrm{mg} \mathrm{kg}^{-1}\right)$ at SRM, and for Zn (70 - $400 \mathrm{mg} \mathrm{kg}^{-1}$ ) at MOJ, CAT, ZUP, SRM, SAB and BEO, while levels above the suggested critical range for plants were measured for: $\mathrm{Cr}\left(>100 \mathrm{mg} \mathrm{kg}^{-1}\right)$ at ZUP, SAB and BEO, Cu (> $\left.125 \mathrm{mg} \mathrm{kg}^{-1}\right)$ at CAT, and $\mathrm{Ni}\left(>100 \mathrm{mg} \mathrm{kg}^{-1}\right)$ at ZUP, SAB and BEO. In addition, a statistically significant difference was found between these sites in terms of the obtained levels of $\mathrm{As}, \mathrm{Cd}, \mathrm{Cr}, \mathrm{Cu}, \mathrm{Ni}, \mathrm{Pb}$ and $\mathrm{Zn}$ (Table 2).

PTE levels in the analysed soil samples were lower than those from our earlier survey on the riparian soil of the River Sava, except for $\mathrm{Cu}$ (Marković et al. 2018). The reason for this is probably due to differences in the analytical methods used for determining element content, as well as the different depths of the riparian soil samples. However, in comparison to the study by Pavlović et al. (2019), the results for $\mathrm{As}, \mathrm{Cd}$ and $\mathrm{Pb}$ were lower or similar, while the measured content of $\mathrm{Cr}, \mathrm{Cu}, \mathrm{Ni}$ and $\mathrm{Zn}$ was higher than previously reported. The differences in the measured element content could be a consequence of water level. Specifically, soil samples in the study conducted by Pavlović et al. (2019) were taken during a high water event, while samples in the present study were taken during a low water event.

When compared to some European rivers with similar anthropogenic pressures (urbanisation, industry, agriculture, etc.), the $\mathrm{As}, \mathrm{Pb}$ and $\mathrm{Zn}$ levels recorded in the riparian soils of the Sava were lower than those in the riparian soils of the Elbe in Germany (Schulz-Zunkel et al. 2013), but higher than in the riparian soils of the Don in Russia (Minkina et al. 2017) and the Kolubara in Serbia (Čakmak et al. 2018). Moreover, the content of $\mathrm{Cu}$ and Ni measured in this study was similar to that in the riparian soils of the Don (Minkina et al. 2017), the Elbe (Schulz-Zunkel et al. 2013) and the Kolubara (Čakmak et al. 2018) rivers. On the other hand, levels of Cd and Cr in the riparian soils at the examined sampling sites were lower than those reported in the riparian soils at all the rivers mentioned above. 
Table 2 PTE levels in riparian soils at the examined sampling sites (in $\mathrm{mg} \mathrm{kg}^{-1}$, with SD in brackets); values in and above the critical range for plants are denoted in bold

\begin{tabular}{|c|c|c|c|c|c|c|c|}
\hline Site & As & Cd & $\mathrm{Cr}$ & $\mathbf{C u}$ & $\mathbf{N i}$ & $\mathbf{P b}$ & Zn \\
\hline MOJ & $3.67(0.46)^{f}$ & $<\operatorname{LoQ}^{d}$ & $15.91(1.07)^{\mathrm{i}}$ & $14.65(0.43)^{g, h}$ & $7.20(0.42)^{1}$ & $60.12(4.23)^{b}$ & $105.67(1.61)^{d}$ \\
\hline RAD & $4.60(0.35)^{f}$ & $<\operatorname{LoQ}^{d}$ & $29.66(0.58)^{e}$ & $21.10(0.08)^{f}$ & $20.71(0.10)^{f}$ & $25.18(0.62)^{f}$ & $58.32(0.54)^{h}$ \\
\hline LIT & $4.84(0.25)^{f}$ & $<\operatorname{LoQ}^{d}$ & $19.52(0.17)^{h}$ & $12.67(0.07)^{\mathrm{h}, \mathrm{i}}$ & $11.86(0.11)^{j}$ & $24.19(0.49)^{f}$ & $47.55(1.72)^{j}$ \\
\hline VRH & $4.45(0.30)^{f}$ & $<\operatorname{LoQ}^{d}$ & $26.76(0.19)^{f}$ & $20.14(0.34)^{f}$ & $16.03(0.05)^{h}$ & $20.07(1.08)^{g}$ & $67.77(0.80)^{g}$ \\
\hline CAT & $7.30(0.42)^{e}$ & $<\operatorname{LoQ}^{d}$ & $31.63(0.19)$ e & $382.26(3.90)^{a}$ & $18.20(0.12)^{g}$ & $27.34(0.78)^{e}$ & $97.30(0.45)^{e}$ \\
\hline ZAG & $4.96(0.32)^{f}$ & $<\operatorname{LoQ}^{d}$ & $16.08(0.12)^{\mathrm{i}}$ & $11.43(0.09)^{\mathrm{i}}$ & $10.04(0.08)^{k}$ & $10.74(0.23)^{i}$ & $41.48(0.33)^{k}$ \\
\hline JAS & $4.37(0.30)^{f}$ & $<\operatorname{LoQ}^{\mathrm{d}}$ & $23.72(0.26)^{g}$ & $12.19(0.16)^{\mathrm{i}}$ & $13.70(0.26)^{i}$ & $12.53(1.38)^{\mathrm{h}, \mathrm{i}}$ & $50.92(0.42)^{j}$ \\
\hline SLB & $7.21(0.21)^{e}$ & $<\operatorname{LoQ}^{d}$ & $43.67(0.40)^{d}$ & $15.62(0.11)^{g}$ & $29.86(0.54)^{\mathrm{e}}$ & $13.06(1.15)^{h}$ & $46.27(0.31)^{\mathrm{i}}$ \\
\hline ZUP & $8.89(0.26)^{d}$ & $<\operatorname{LoQ}^{d}$ & $123.88(0.69)^{a}$ & $30.28(0.14)^{e}$ & $101.07(1.28)^{c}$ & $25.60(0.89)^{e, f}$ & $87.70(0.30)^{f}$ \\
\hline SRM & $13.93(0.21)^{c}$ & $0.12(0.00)^{c}$ & $99.46(1.54)^{c}$ & $32.77(0.27)^{d}$ & $81.17(0.21)^{d}$ & $30.46(0.71)^{d}$ & $114.47(0.73)^{c}$ \\
\hline SAB & $16.54(0.29)^{b}$ & $0.14(0.00)^{b}$ & $123.38(2.25)^{\mathrm{a}}$ & $42.55(0.14)^{c}$ & $113.37(1.25)^{a}$ & $44.07(0.58)^{c}$ & $159.03(2.00)^{b}$ \\
\hline BEO & $21.86(0.30)^{a}$ & $0.72(0.00)^{a}$ & $119.22(2.04)^{b}$ & $58.52(0.18)^{b}$ & $106.16(0.40)^{b}$ & $74.40(1.11)^{a}$ & $223.38(1.09)^{a}$ \\
\hline Average & 8.55 & 0.33 & 56.07 & 54.52 & 44.11 & 30.65 & 87.19 \\
\hline $\begin{array}{l}\text { Average range } \\
\text { in world soils }\end{array}$ & $4.4-8.4$ & $0.37-0.45$ & $47-51$ & $13-23$ & $13-26$ & $22-28$ & $45-60$ \\
\hline $\begin{array}{l}\text { Background for } \\
\text { European soils }\end{array}$ & - & $1-3$ & $50-100$ & $50-140$ & $30-75$ & $50-300$ & $150-300$ \\
\hline $\begin{array}{l}\text { Background values } \\
\text { for the study area }\end{array}$ & 11.53 & 0.68 & 72.52 & 24.12 & 41.33 & 44.03 & 91.64 \\
\hline $\begin{array}{l}\text { Critical range } \\
\text { for plants }\end{array}$ & $20-50$ & $>2.5$ & $75-100$ & $60-125$ & $>100$ & $>100$ & $70-400$ \\
\hline
\end{tabular}

Reference values for element content in soil: ${ }^{\mathrm{a}}$ Kabata-Pendias, 2011; ${ }^{\mathrm{b}}$ Gawlik and Bidoglio 2006; ${ }^{\mathrm{c}}$ Marković et al. 2018; ${ }^{\mathrm{d}}$ Alloway 2013

$<$ LoQ - below the Limit of Quantification

Values with the same letter were not significantly different. 
Enrichment factor

In order to assess pollution in soils in terms of the sources of PTEs, the enrichment factor (EF) was calculated and the results are presented in Table 3. Calculating the soil enrichment factor is important given the fact that PTEs can present a risk to vegetation and, consequently, the living world through food chains (Chojnacka et al. 2005). The results revealed deficiency to minimal enrichment $(\mathrm{EF}<2)$ for most elements at most of the sampling sites. Deficiency to minimal enrichment in soil could indicate that the origin of PTEs is predominantly natural (Mil-Homens et al. 2006). Moderate enrichment (EF 2 - 5) was found for $\mathrm{Cu}$ at MOJ and RAD, for Ni at ZUP, SRM, SAB and BEO, and for Zn at MOJ and CAT (Table 3). Significant enrichment was calculated for Pb at $\mathrm{MOJ}(\mathrm{EF}=5.60)$, while $\mathrm{Cu}$ enrichment at CAT was very high $(\mathrm{EF}=33.41)$. Soil enrichment with $\mathrm{Pb}$ and $\mathrm{Zn}$ at MOJ is most likely due to the chemical weathering of parent rock and ore deposits (Marković et al. 2018; Pavlović et al. 2019). Furthermore, enrichment with $\mathrm{Pb}, \mathrm{Zn}$ and $\mathrm{Cu}$ in the upper stretch of the river could be related to anthropogenic sources, such as mining or the application of different types of fertilizers (Adriano 2001; Alloway 2013). Very high enrichment with $\mathrm{Cu}$ in the riparian soils at CAT probably occurs due to agricultural (the application of grapevine fungicides that contain $\mathrm{Cu}$; Fan et al. 2011) and industrial activities (the paper and wood processing industry; ISRBC 2016, Marković et al. 2018). In the lower stretch of the river, soils are moderately enriched with $\mathrm{Ni}$, but this elevated EF is predominantly a result of the geological substrate (Zovko and Romić 2011; Pavlović et al. 2019) and, to some extent, different anthropogenic activities (Milačič et al. 2017; Marković et al. 2018).

Table 3 Enrichment factors (EF) for PTEs in soils at selected sampling sites

\begin{tabular}{crrrrrrr}
\hline & $\mathbf{E F}_{\text {As }}$ & $\mathbf{E F}$ Cd & $\mathbf{E F}_{\mathbf{C r}}$ & $\mathbf{E F}_{\mathbf{C u}}$ & $\mathbf{E F}_{\mathbf{N i}}$ & $\mathbf{E F}_{\mathbf{P b}}$ & $\mathbf{E F}_{\mathbf{Z n}}$ \\
\hline MOJ & 1.31 & 0.00 & 0.90 & $\mathbf{2 . 4 9}$ & 0.71 & $\mathbf{5 . 6 0}$ & $\mathbf{4 . 7 3}$ \\
RAD & 1.04 & 0.00 & 1.06 & $\mathbf{2 . 2 7}$ & 1.30 & 1.49 & 1.65 \\
LIT & 1.40 & 0.00 & 0.90 & 1.75 & 0.95 & 1.83 & 1.73 \\
VRH & 0.85 & 0.00 & 0.81 & 1.84 & 0.85 & 1.00 & 1.63 \\
CAT & 1.33 & 0.00 & 0.92 & $\mathbf{3 3 . 4 1}$ & 0.93 & 1.31 & $\mathbf{2 . 2 4}$ \\
ZAG & 1.22 & 0.00 & 0.63 & 1.34 & 0.69 & 0.69 & 1.28 \\
JAS & 0.90 & 0.00 & 0.77 & 1.20 & 0.78 & 0.67 & 1.31 \\
SLB & 0.78 & 0.00 & 0.75 & 0.81 & 0.90 & 0.37 & 0.63 \\
ZUP & 0.81 & 0.00 & 1.80 & 1.32 & $\mathbf{2 . 5 8}$ & 0.61 & 1.01 \\
SRM & 1.31 & 0.19 & 1.49 & 1.47 & $\mathbf{2 . 1 3}$ & 0.75 & 1.35 \\
SAB & 1.14 & 0.16 & 1.36 & 1.41 & $\mathbf{2 . 1 9}$ & 0.80 & 1.38 \\
BEO & 1.48 & 0.82 & 1.28 & 1.89 & $\mathbf{2 . 0 0}$ & 1.32 & 1.44 \\
\hline
\end{tabular}

EFs $>2$ are denoted in bold

PTEs in plants

PTE levels in the roots and leaves of the examined plants are presented in Tables 4 and 5. Those that are considered to be toxic for plants (Marschner 1995; Pugh et al. 2002; Kabata-Pendias 2011; Alloway 2013) are denoted in bold. The trend for the average element content in roots was as follows: $\mathrm{Zn}>\mathrm{Cu}>\mathrm{Cd}>\mathrm{Ni}>\mathrm{Pb}>\mathrm{Cr}$ $>$ As in S. alba; $\mathrm{Zn}>\mathrm{Cr}>\mathrm{Cu}>\mathrm{Pb}>\mathrm{As}>\mathrm{Ni}>\mathrm{Cd}$ in J. regia; and $\mathrm{Zn}>\mathrm{Cu}>\mathrm{Cr}>\mathrm{Ni}>\mathrm{Pb}>\mathrm{As}>\mathrm{Cd}$ in $\mathrm{P}$. nigra. In leaves, the trend was as follows: $\mathrm{Zn}>\mathrm{Cu}>\mathrm{Ni}>\mathrm{Cr}>\mathrm{Cd}>\mathrm{Pb}>\mathrm{As}$ in S. alba; $\mathrm{Zn}>\mathrm{Cu}>\mathrm{As}>\mathrm{Cr}>\mathrm{Pb}$ $>\mathrm{Ni}>\mathrm{Cd}$ in J. regia; and $\mathrm{Zn}>\mathrm{Cu}>\mathrm{As}>\mathrm{Cd}>\mathrm{Pb}>\mathrm{Ni}>\mathrm{Cr}$ in P. nigra. 
Copper and Zn are essential elements in plants, which is why they are the most abundant elements in samples. However, in some samples their concentrations were very low, especially in the roots and leaves of $J$. regia and $P$. nigra. In terms of $\mathrm{Cu}$, the roots of some species have the ability to retain it and to prevent its transport to shoots. The levels of other elements in plants, combined with the low mobility of $\mathrm{Cu}$, could lead to $\mathrm{Cu}$ deficiency in plant leaves (Kabata-Pendias 2011). Additionally, the binding of $\mathrm{Cu}$ by soils is highly dependent on soil pH, which is why $\mathrm{Cu}$ availability decreases at about pH 7-8 (Kabata-Pendias 2011). Since the study by Pavlović et al. (2019) reported alkaline $\mathrm{pH}$ for this research area, this could be the reason for $\mathrm{Cu}$ deficiency in the analysed plants. As for Zn, its availability is highly dependent on clay content in the soil; thus the low $\mathrm{Zn}$ accumulation in the leaves of $J$. regia may be a result of this as soils in our research area contain up to $35 \%$ clay (Pavlović et al. 2019). 
Table 4 Potentially toxic elements in roots, presented as mean values with SD in brackets, in mg kg $^{-1}$ on a dry mass basis

\begin{tabular}{|c|c|c|c|c|c|c|c|c|}
\hline Species & Site & As & Cd & $\mathrm{Cr}$ & $\mathbf{C u}$ & $\mathbf{N i}$ & $\mathbf{P b}$ & Zn \\
\hline \multirow{13}{*}{ 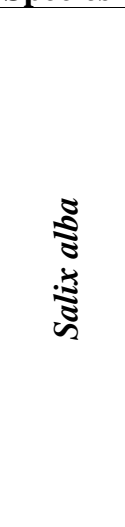 } & MOJ & $2.05(0.51)^{f, g}$ & $0.22(0.00)^{\mathrm{e}}$ & $<\operatorname{LoQ}^{\mathrm{e}}$ & $2.28(0.00)^{g}$ & $<\operatorname{LoQ}^{f}$ & $2.19(0.12)^{\mathrm{e}, \mathrm{f}, \mathrm{g}}$ & $36.99(0.48)^{\mathrm{e}}$ \\
\hline & RAD & $3.19(0.57)^{d, e}$ & $0.22(0.00)^{e}$ & $<\operatorname{LoQ}^{\mathrm{e}}$ & $2.66(0.00)^{g}$ & $<\operatorname{LoQ}^{\mathrm{f}}$ & $2.33(0.26)^{d, e, f}$ & $18.35(0.25)^{g}$ \\
\hline & LIT & $2.42(0.43)^{e, f}$ & $0.22(0.00)^{e}$ & $<\operatorname{LoQ}^{\mathrm{e}}$ & $5.19(0.07)^{f}$ & $1.83(0.26)^{\mathrm{d}, \mathrm{e}}$ & $3.14(0.13)^{d}$ & $20.29(1.15)^{g}$ \\
\hline & VRH & $1.33(0.52)^{g}$ & $0.25(0.00)^{e}$ & $0.64(0.07)^{\mathrm{e}}$ & $7.41(2.59){ }^{e}$ & $0.27(0.08)^{f}$ & $2.19(0.07)^{e, f, g}$ & $33.17(1.75)^{e}$ \\
\hline & CAT & $2.78(0.33)^{e, f}$ & $0.44(0.00)^{d, e}$ & $1.00(0.00)^{\mathrm{d}, \mathrm{e}}$ & $6.50(0.00)^{e, f}$ & $0.55(0.14)^{e, f}$ & $1.83(0.15)^{\mathrm{f}, \mathrm{g}}$ & $53.07(0.33)^{c, d}$ \\
\hline & ZAG & $<\mathrm{LoQ}^{\mathrm{h}}$ & $0.25(0.00)^{\mathrm{e}}$ & $1.33(0.00)^{\mathrm{d}, \mathrm{e}}$ & $7.33(1.28)^{\text {e }}$ & $0.61(0.14)^{e, f}$ & $2.22(0.09)^{\mathrm{d}, \mathrm{e}, \mathrm{f}, \mathrm{g}}$ & $48.89(2.16)^{d}$ \\
\hline & JAS & $2.58(0.31)^{e, f}$ & $0.44(0.00)^{\mathrm{d}, \mathrm{e}}$ & $0.58(0.09)^{\mathrm{e}}$ & $5.28(0.25)^{f}$ & $0.39(0.09)^{\mathrm{e}, \mathrm{f}}$ & $1.33(0.21)^{g}$ & $26.59(0.11)^{f}$ \\
\hline & SLB & $3.86(0.81)^{c, d}$ & $0.67(0.00)^{d}$ & $2.61(0.31)^{d}$ & $7.75(0.09)^{\mathrm{e}}$ & $2.70(0.34)^{d}$ & $2.95(0.52)$ d,e & $35.82(2.97)^{\mathrm{e}}$ \\
\hline & ZUP & $4.72(0.45)^{b, c}$ & $1.22(0.12)^{c}$ & $14.57(2.96)^{a}$ & $14.58(1.09)^{d}$ & $19.08(2.40)^{a}$ & $7.36(1.29)^{b}$ & $48.37(1.81)^{d}$ \\
\hline & SRM & $5.53(0.13)^{b}$ & $1.33(0.00)^{c}$ & $5.55(0.61)^{c}$ & $17.44(0.62)^{c}$ & $10.47(1.13)^{b}$ & $5.50(0.30)^{c}$ & $56.72(1.29)^{\mathrm{c}}$ \\
\hline & SAB & $6.42(0.25)^{a}$ & $4.48(0.09)^{b}$ & $7.25(0.82) b, c$ & $22.14(0.58)^{b}$ & $10.17(0.49)^{b}$ & $9.89(0.49)^{\mathrm{a}}$ & $125.55(6.55)^{b}$ \\
\hline & BEO & $5.50(0.23)^{b}$ & $86.96(0.63)^{a}$ & $7.65(0.57)^{b}$ & $26.46(0.41)^{a}$ & $8.16(0.14)^{\mathrm{c}}$ & $10.48(0.46)^{\mathrm{a}}$ & $278.18(1.71)^{a}$ \\
\hline & Average & 3.37 & 8.06 & 3.43 & 10.42 & 4.52 & 4.28 & 65.17 \\
\hline \multirow{8}{*}{ 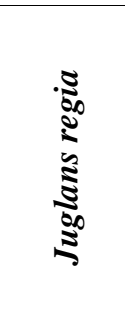 } & MOJ & $2.99(0.43)^{b, c}$ & $<\operatorname{LoQ}^{\mathrm{c}}$ & $6.17(0.24)^{c, d}$ & $4.75(1.55)^{c}$ & $0.24(0.08)^{\mathrm{e}}$ & $6.07(0.27)^{a}$ & $26.21(1.72)^{\mathrm{c}}$ \\
\hline & RAD & $2.12(0.66)^{c, d}$ & $<\operatorname{LoQ}^{\mathrm{c}}$ & $6.80(0.29)^{c}$ & $8.27(0.15)^{b}$ & $1.14(0.10)^{d}$ & $1.62(0.23)^{\mathrm{e}}$ & $25.19(0.67)^{\mathrm{c}}$ \\
\hline & LIT & $2.48(0.09)^{b, c, d}$ & $<\operatorname{LoQ}^{c}$ & $6.00(0.06)^{c, d}$ & $7.96(0.04)^{b}$ & $0.77(0.01)^{d}$ & $1.26(0.03) \mathrm{e}$ & $27.70(0.23)^{b}$ \\
\hline & VRH & $1.95(0.65)^{d}$ & $<\operatorname{LoQ}^{\mathrm{c}}$ & $4.96(0.53)^{d}$ & $1.92(0.46)^{\mathrm{e}}$ & $<\mathrm{LoQ}^{\mathrm{e}}$ & $1.41(0.12)^{\mathrm{e}}$ & $1.60(0.00)^{f}$ \\
\hline & CAT & $3.31(0.55)^{b}$ & $0.25(0.00)^{b}$ & $24.37(1.67)^{a}$ & $7.20(0.17)^{b}$ & $3.53(0.55)^{b}$ & $4.91(0.47)^{b}$ & $18.78(0.47)^{d}$ \\
\hline & JAS & $4.91(0.80)^{a}$ & $0.25(0.00)^{b}$ & $12.24(1.67)^{b}$ & $3.53(0.54)^{d}$ & $2.22(0.18)^{c}$ & $2.89(0.18)^{d}$ & $8.12(0.62)^{\mathrm{e}}$ \\
\hline & SRM & $1.62(0.37)^{d}$ & $0.45(0.24)^{\mathrm{a}}$ & $11.50(0.55)^{b}$ & $10.05(0.35)^{a}$ & $7.66(0.22)^{a}$ & $3.57(0.51)^{c}$ & $45.19(0.31)^{\mathrm{a}}$ \\
\hline & Average & 2.77 & 0.14 & 10.29 & 6.24 & 2.22 & 3.10 & 21.83 \\
\hline \multirow{10}{*}{ 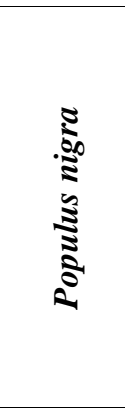 } & MOJ & $2.12(0.44)^{b}$ & $0.26(0.00)^{\mathrm{e}}$ & $5.34(1.11)^{b}$ & $10.33(0.86)^{a}$ & $2.46(0.47)^{\mathrm{e}}$ & $4.04(0.26)^{a}$ & $19.67(2.17)^{\mathrm{e}}$ \\
\hline & VRH & $5.38(0.17)^{a}$ & $0.26(0.00)^{e}$ & $4.22(1.30)^{b, c}$ & $5.84(0.55)^{\mathrm{e}}$ & $0.75(0.38)^{g}$ & $1.56(0.69)^{\mathrm{d}, \mathrm{e}}$ & $11.05(0.86)^{f}$ \\
\hline & CAT & $5.19(0.24)^{a}$ & $0.30(0.11)^{\mathrm{d}, \mathrm{e}}$ & $3.90(0.00)^{\mathrm{c}}$ & $4.03(0.07)^{f}$ & $0.75(0.11)^{g}$ & $3.04(0.61)^{b}$ & $25.54(0.26)^{d}$ \\
\hline & ZAG & $<\mathrm{LoQ}^{\mathrm{c}}$ & $0.69(0.13)^{b}$ & $2.27(0.12)^{\mathrm{e}}$ & $7.08(0.09)^{d}$ & $0.96(0.11)^{g}$ & $1.23(0.23)^{\mathrm{e}}$ & $49.68(0.46)^{c}$ \\
\hline & JAS & $<\operatorname{LoQ}^{\mathrm{c}}$ & $0.69(0.13)^{b}$ & $3.93(0.21)^{c}$ & $5.25(0.09)^{\mathrm{e}}$ & $1.50(0.11)^{f}$ & $1.41(0.18)^{\mathrm{d}, \mathrm{e}}$ & $61.16(4.26)^{b}$ \\
\hline & ZUP & $<\operatorname{LoQ}^{\mathrm{c}}$ & $0.43(0.13)^{\mathrm{c}, \mathrm{d}}$ & $2.60(0.00)^{\mathrm{d}, \mathrm{e}}$ & $8.50(0.15)^{c}$ & $4.71(0.00)^{d}$ & $1.26(0.23)^{\mathrm{e}}$ & $27.19(0.43)^{d}$ \\
\hline & SRM & $<\operatorname{LoQ}^{\mathrm{c}}$ & $0.52(0.00)^{\mathrm{c}}$ & $4.75(0.00)^{b, c}$ & $7.40(0.08)^{d}$ & $6.34(0.22)^{b}$ & $2.59(0.28)^{b, c}$ & $25.81(0.52)^{d}$ \\
\hline & SAB & $<\operatorname{LoQ}^{\mathrm{c}}$ & $1.03(0.00)^{\mathrm{a}}$ & $7.35(0.79)^{a}$ & $9.79(0.00)^{b}$ & $7.94(0.08)^{a}$ & $4.33(0.39)^{\mathrm{a}}$ & $53.11(3.99)^{c}$ \\
\hline & BEO & $<\operatorname{LoQ}^{\mathrm{c}}$ & $0.52(0.00)^{c}$ & $3.61(0.56)^{c, d}$ & $8.45(0.13)^{c}$ & $5.36(0.08)^{c}$ & $2.11(0.23)^{c, d}$ & $85.93(1.73)^{a}$ \\
\hline & Average & 1.41 & 0.52 & 4.22 & 7.41 & 3.42 & 2.40 & 39.90 \\
\hline \multicolumn{2}{|c|}{ Deficit } & - & - & - & $2-5^{a}$ & - & - & $10-20^{\mathrm{a}}$ \\
\hline \multirow{2}{*}{\multicolumn{2}{|c|}{$\begin{array}{c}\text { Normal range } \\
\text { Toxic range }\end{array}$}} & $1-1.7^{a}$ & $0.002-1^{\mathrm{a}}$ & $0.1-0.5^{\mathrm{a}}$ & $5-30^{\mathrm{a}, \mathrm{c}}$ & $0.1-5^{\mathrm{a}, \mathrm{b}}$ & $0.2-10^{\mathrm{a}, \mathrm{c}}$ & $27-150^{\mathrm{a}, \mathrm{d}}$ \\
\hline & & $5-20^{\mathrm{a}, \mathrm{b}}$ & $5-30^{a, b}$ & $5-30^{a, b}$ & $20-100^{\mathrm{a}, \mathrm{c}}$ & $10-100^{\mathrm{a}, \mathrm{b}}$ & $30-300^{\mathrm{a}, \mathrm{b}}$ & $100-400^{\mathrm{a}, \mathrm{b}, \mathrm{d}}$ \\
\hline
\end{tabular}

Values for deficit, normal and toxic range: ${ }^{\mathrm{a}}$ Kabata-Pendias 2011; ${ }^{\mathrm{b}}$ Alloway 2013; ${ }^{\mathrm{c}}$ Pugh et al. 2002; ${ }^{\mathrm{d}}$ Marschner 1995; < LoQ - below the Limit of Quantification

Values with the same letter were not significantly different. 
Table 5 Potentially toxic elements in leaves, presented as mean values with SD in brackets, in $\mathrm{mg} \mathrm{kg}^{-1}$ on a dry mass basis

\begin{tabular}{|c|c|c|c|c|c|c|c|c|}
\hline Species & Site & As & $\mathbf{C d}$ & $\mathrm{Cr}$ & $\mathrm{Cu}$ & $\mathbf{N i}$ & $\mathbf{P b}$ & Zn \\
\hline \multirow{13}{*}{ 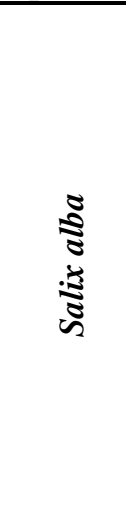 } & MOJ & $3.06(0.46)^{b}$ & $0.44(0.00)^{\mathrm{e}}$ & $<\operatorname{LoQ}^{d}$ & $4.92(0.09)^{f}$ & $0.44(0.09)^{e, f}$ & $<\operatorname{LoQ}^{\mathrm{c}}$ & $149.61(0.59)^{f}$ \\
\hline & RAD & $<\operatorname{LoQ}^{\mathrm{d}}$ & $0.22(0.00)^{f}$ & $<\operatorname{LoQ}^{d}$ & $5.11(0.09)^{f}$ & $<\operatorname{LoQ}^{\mathrm{f}}$ & $<\operatorname{LoQ}^{\mathrm{c}}$ & $157.07(0.28)^{e}$ \\
\hline & LIT & $<\mathrm{LoQ}^{\mathrm{d}}$ & $0.22(0.00)^{f}$ & $0.44(0.09)^{\mathrm{c}}$ & $6.31(0.22)^{\mathrm{e}}$ & $0.42(0.09)$ e,f & $0.71(0.06)^{b}$ & $44.41(1.00)^{1}$ \\
\hline & VRH & $<\operatorname{LoQ}^{d}$ & $1.55(0.00)^{b}$ & $<\mathrm{LoQ}^{\mathrm{d}}$ & $5.05(0.14)^{f}$ & $<\operatorname{LoQ}^{\mathrm{f}}$ & $<\mathrm{LoQ}^{\mathrm{c}}$ & $227.91(2.24)^{b}$ \\
\hline & CAT & $<\operatorname{LoQ}^{\mathrm{d}}$ & $5.13(0.09)^{a}$ & $<\operatorname{LoQ}^{\mathrm{d}}$ & $6.28(0.31)^{e}$ & $<\operatorname{LoQ}^{\mathrm{f}}$ & $<\operatorname{LoQ}^{\mathrm{c}}$ & $308.03(2.67)^{a}$ \\
\hline & ZAG & $<\operatorname{LoQ}^{d}$ & $0.63(0.09)^{d}$ & $<\operatorname{LoQ}^{d}$ & $5.33(0.18)^{f}$ & $<\operatorname{LoQ}^{\mathrm{f}}$ & $<\operatorname{LoQ}^{\mathrm{c}}$ & $83.48(0.48)^{k}$ \\
\hline & JAS & $<\operatorname{LoQ}^{\mathrm{d}}$ & $0.22(0.00)^{f}$ & $<\operatorname{LoQ}^{\mathrm{d}}$ & $7.96(0.07)^{c}$ & $0.50(0.00)^{\mathrm{e}}$ & $<\operatorname{LoQ}^{\mathrm{c}}$ & $102.08(1.94)^{j}$ \\
\hline & SLB & $<\operatorname{LoQ}^{d}$ & $0.66(0.00)^{d}$ & $2.39(0.31)^{b}$ & $6.83(0.37)^{d}$ & $1.42(0.09)^{d}$ & $1.03(0.19)^{b}$ & $133.30(2.50)^{g}$ \\
\hline & ZUP & $1.07(0.23)^{c}$ & $0.66(0.00)^{d}$ & $2.58(0.09)^{b}$ & $8.00(0.18)^{c}$ & $4.14(0.07)^{b}$ & $1.00(0.24)^{b}$ & $105.34(0.69)^{i}$ \\
\hline & SRM & $<\operatorname{LoQ}^{\mathrm{d}}$ & $0.66(0.00)^{d}$ & $<\operatorname{LoQ}^{\mathrm{d}}$ & $6.17(0.61)$ d,e & $<\operatorname{LoQ}^{\mathrm{f}}$ & $<\mathrm{LoQ}^{\mathrm{c}}$ & $175.41(0.44)^{d}$ \\
\hline & SAB & $6.31(0.59)^{\mathrm{a}}$ & $1.33(0.00)^{\mathrm{c}}$ & $11.48(0.60)^{a}$ & $11.34(0.21)^{\mathrm{a}}$ & $13.26(0.84)^{a}$ & $8.34(0.67)^{a}$ & $204.55(2.17)^{c}$ \\
\hline & BEO & $<\mathrm{LoQ}^{\mathrm{d}}$ & $0.44(0.00)^{e}$ & $<\operatorname{LoQ}^{d}$ & $10.39(0.25)^{b}$ & $2.33(0.00)^{\mathrm{c}}$ & $<\mathrm{LoQ}^{\mathrm{c}}$ & $127.52(0.39)^{h}$ \\
\hline & Average & 0.87 & 1.01 & 1.41 & 6.97 & 1.88 & 0.92 & 151.56 \\
\hline \multirow{8}{*}{ 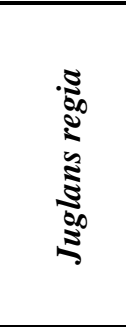 } & MOJ & $1.39(0.12)^{b}$ & $<\mathrm{LoQ}$ & $1.43(0.84)^{c}$ & $6.53(0.52)^{b}$ & $<\mathrm{LoQ}^{\mathrm{d}}$ & $1.34(0.32)^{\mathrm{a}, \mathrm{b}}$ & $19.66(0.23)^{c}$ \\
\hline & RAD & $1.71(0.14)^{b}$ & $<\operatorname{LoQ}$ & $2.20(0.24)^{b}$ & $9.64(0.20)^{\mathrm{a}}$ & $0.44(0.08)^{\mathrm{c}}$ & $1.11(0.18)^{\mathrm{a}, \mathrm{b}}$ & $22.74(0.54)^{b}$ \\
\hline & LIT & $5.26(0.63)^{a}$ & $<\mathrm{LoQ}$ & $1.18(0.18)^{c, d}$ & $5.19(0.07)^{c}$ & $0.44(0.08)^{c}$ & $<\operatorname{LoQ}^{\mathrm{c}}$ & $25.55(0.11)^{a}$ \\
\hline & VRH & $5.69(0.22)^{a}$ & $<\mathrm{LoQ}$ & $1.10(0.20)^{c, d}$ & $4.33(0.18)^{d}$ & $<\operatorname{LoQ}^{\mathrm{d}}$ & $<\operatorname{LoQ}^{\mathrm{c}}$ & $1.60(0.00)^{\mathrm{f}}$ \\
\hline & CAT & $5.59(0.85)^{a}$ & $<$ LoQ & $0.62(0.09)^{d}$ & $3.44(0.14)^{e}$ & $<\operatorname{LoQ}^{\mathrm{d}}$ & $<\operatorname{LoQ}^{\mathrm{c}}$ & $8.32(0.12)^{e}$ \\
\hline & JAS & $1.54(0.55)^{b}$ & $<\operatorname{LoQ}$ & $4.63(0.00)^{a}$ & $4.75(0.09)^{d}$ & $0.94(0.10)^{b}$ & $1.44(0.33)^{\mathrm{a}}$ & $8.24(0.00)^{e}$ \\
\hline & SRM & $2.10(1.13)^{b}$ & $<\mathrm{LoQ}$ & $1.62(0.18)^{b, c}$ & $4.33(0.18)^{d}$ & $1.55(0.10)^{\mathrm{a}}$ & $1.09(0.14)^{b}$ & $12.74(0.12)^{d}$ \\
\hline & Average & 3.33 & 1 & 1.83 & 5.46 & 0.48 & 0.71 & 14.12 \\
\hline \multirow{10}{*}{ 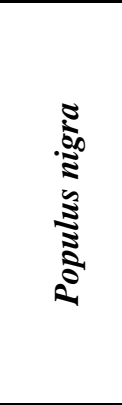 } & MOJ & $<\operatorname{LoQ}^{\mathrm{c}}$ & $<\mathrm{LoQ}^{\mathrm{g}}$ & $<\mathrm{LoQ}^{\mathrm{c}}$ & $5.62(0.38)^{c}$ & $<\mathrm{LoQ}^{\mathrm{d}}$ & $0.73(0.29)^{b}$ & $42.88(4.74)^{g}$ \\
\hline & VRH & $<\operatorname{LoQ}^{\mathrm{c}}$ & $<\operatorname{LoQ}^{\mathrm{g}}$ & $0.22(0.00)^{b}$ & $6.79(0.17)^{a}$ & $<\operatorname{LoQ}^{\mathrm{d}}$ & $0.28(0.09)^{b}$ & $24.08(1.88)^{h}$ \\
\hline & CAT & $<\operatorname{LoQ}^{\mathrm{c}}$ & $2.06(0.00)^{\mathrm{a}}$ & $0.22(0.00)^{b}$ & $4.78(0.08)^{d}$ & $0.20(0.00)^{c}$ & $3.15(1.12)^{\mathrm{a}}$ & $135.23(0.68)^{b}$ \\
\hline & ZAG & $<\operatorname{LoQ}^{\mathrm{c}}$ & $1.29(0.00)^{c}$ & $<\operatorname{LoQ}^{\mathrm{c}}$ & $4.36(0.16)^{e}$ & $<\operatorname{LoQ}^{\mathrm{d}}$ & $0.30(0.08)^{b}$ & $122.00(1.05)^{c}$ \\
\hline & JAS & $2.56(0.23)^{\mathrm{a}, \mathrm{b}}$ & $1.03(0.00)^{d}$ & $0.32(0.12)^{a}$ & $7.06(0.14)^{a}$ & $0.27(0.11)^{c}$ & $0.30(0.08)^{b}$ & $74.59(0.34)^{\mathrm{e}}$ \\
\hline & ZUP & $2.40(0.89)^{\mathrm{a}, \mathrm{b}}$ & $1.29(0.00)^{c}$ & $<\operatorname{LoQ}^{\mathrm{c}}$ & $6.27(0.13)^{b}$ & $2.04(0.13)^{a}$ & $<\mathrm{LoQ}^{\mathrm{b}}$ & $94.73(0.97)^{d}$ \\
\hline & SRM & $2.82(0.94)^{\mathrm{a}}$ & $0.52(0.00)^{f}$ & $<\operatorname{LoQ}^{\mathrm{c}}$ & $4.17(0.00)^{e}$ & $0.20(0.00)^{c}$ & $<\mathrm{LoQ}^{\mathrm{b}}$ & $10.43(0.89)^{i}$ \\
\hline & SAB & $1.76(0.28)^{b}$ & $1.55(0.00)^{b}$ & $<\operatorname{LoQ}^{\mathrm{c}}$ & $6.03(0.16)^{b}$ & $0.51(0.00)^{b}$ & $<\operatorname{LoQ}^{\mathrm{b}}$ & $207.77(3.54)^{a}$ \\
\hline & BEO & $3.17(0.49)^{\mathrm{a}}$ & $0.77(0.00)^{\mathrm{e}}$ & $<\operatorname{LoQ}^{\mathrm{c}}$ & $4.06(0.08)^{e}$ & $<\mathrm{LoQ}^{\mathrm{d}}$ & $<\mathrm{LoQ}^{\mathrm{b}}$ & $50.93(1.21)^{f}$ \\
\hline & Average & 1.41 & 0.95 & 0.08 & 5.46 & 0.36 & 0.53 & 84.74 \\
\hline \multirow{3}{*}{\multicolumn{2}{|c|}{$\begin{array}{l}\text { Deficit } \\
\text { ormal range } \\
\text { oxic range }\end{array}$}} & - & - & - & $2-5^{a}$ & - & - & $10-20^{\mathrm{a}}$ \\
\hline & & $1-1.7^{\mathrm{a}}$ & $0.002-1^{\mathrm{a}}$ & $0.1-0.5^{\mathrm{a}}$ & $5-30^{\mathrm{a}, \mathrm{c}}$ & $0.1-5^{\mathrm{a}, \mathrm{b}}$ & $0.2-10^{\mathrm{a}, \mathrm{c}}$ & $27-150^{\mathrm{a}, \mathrm{d}}$ \\
\hline & & $5-20^{\mathrm{a}, \mathrm{b}}$ & $5-30^{\mathrm{a}, \mathrm{b}}$ & $5-30^{\mathrm{a}, \mathrm{b}}$ & $20-100^{\mathrm{a}, \mathrm{c}}$ & $10-100^{\mathrm{a}, \mathrm{b}}$ & $30-300^{\mathrm{a}, \mathrm{b}}$ & $100-400^{\mathrm{a}, \mathrm{b}, \mathrm{d}}$ \\
\hline
\end{tabular}

Values for deficit, normal and toxic range: ${ }^{\text {a }}$ Kabata-Pendias 2011; ${ }^{\mathrm{b}}$ Alloway 2013; ${ }^{\mathrm{c}}$ Pugh et al. 2002; ${ }^{\mathrm{d}}$ Marschner 1995; < LoQ - below the Limit of Quantification Values with the same letter were not significantly different. 


\section{PTEs in Salix alba}

Salix alba accumulated various PTEs in its roots at levels considered toxic for plants: As (5 - $20 \mathrm{mg} \mathrm{kg}^{-1}$ ) at SRM, SAB and BEO, Cd (5 - $\left.30 \mathrm{mg} \mathrm{kg}^{-1}\right)$ at BEO, Cr (5 - $\left.30 \mathrm{mg} \mathrm{kg}^{-1}\right)$ at ZUP, SRM, SAB and BEO, Ni (10 - $\left.100 \mathrm{mg} \mathrm{kg}^{-1}\right)$ at ZUP, SRM and SAB, and Zn (100 - $\left.400 \mathrm{mg} \mathrm{kg}^{-1}\right)$ at SAB and BEO (Marschner 1995; Pugh et al. 2002; Kabata-Pendias 2011; Alloway 2013). Statistically significant differences between the obtained values of $\mathrm{Cd}, \mathrm{Cr}, \mathrm{Ni}$ and $\mathrm{Zn}$ in roots were found between the investigated stands (Table 4). On the other hand, there was a deficit of $\mathrm{Cu}\left(2-5 \mathrm{mg} \mathrm{kg}^{-1}\right)$ measured at MOJ and RAD and of Zn (10 - $\left.20 \mathrm{mg} \mathrm{kg}^{-1}\right)$ at RAD (Kabata-Pendias 2011). In S. alba leaves, toxic levels were measured for As (6.31 mg kg-1), Cr (11.48 mg kg-1) and Ni (13.26 mg kg-1) at SAB, Cd (5.13 $\left.\mathrm{mg} \mathrm{kg}^{-1}\right)$ at CAT, and Zn at all sampling sites except LIT and ZAG (Table 5). All the sites mentioned above differ from one another in terms of the obtained content of As, Cd, Cr, $\mathrm{Ni}$ and Zn in S. alba leaves (Table 5).

The results from this study show that S. alba is a good accumulator of Cd and Zn, a fact supported by numerous previous studies (Vandecasteele et al. 2002; Vysloužilová et al. 2003; Dos Santos Utmazian et al. 2007; Bedell et al. 2009; Han et al. 2013). In S. alba roots at BEO, besides toxic levels of Cd and Zn, the content of other elements was elevated, too, or even at toxic levels for plants, which might be connected to specific point sources of pollution at this sampling site, leading to the release of PTEs in a form that is more available for plant uptake (Pavlović et al. 2019).

The results obtained in our research in terms of the content of the analysed PTEs in the leaves of $S$. alba are similar to those from earlier research undertaken along the River Danube (Pavlović et al. 2016). On the other hand, in a study conducted by Zimmer et al. (2012) on the floodplains of the River Elbe, while As, $\mathrm{Cu}, \mathrm{Ni}$ and $\mathrm{Pb}$ levels in Salix spp. leaves were similar to those in our present study, Cd and Zn content was much higher. The lower uptake of Cd and Zn in our study is probably due to the lower PTE content in soils compared to the riparian soils of the Elbe, as well as due to the characteristics of the Salix hybrid clone that Zimmer et al. (2012) used for analysis. Vandecasteele et al. (2002) also reported higher levels of $\mathrm{Zn}$ in S. alba leaves and roots at polluted dredged sediment disposal sites along the Scheldt and Leie rivers in Belgium, while the content of Cd in leaves was similar to that recorded in the present study. Meers et al. (2003) also conducted research on different Salix sp. clones growing at dredged sediment disposal sites on the River Leie, where higher levels of Cd and $\mathrm{Zn}$ were recorded, while $\mathrm{Cu}, \mathrm{Ni}$ and $\mathrm{Pb}$ content in leaves was similar to the present study. As in the case of the Elbe, a large area along the River Leie is also affected by PTE pollution, so a higher uptake of elements in Salix sp. leaves is possible, but it is also dependent on the characteristics of the Salix clones used in the research. In soils experimentally enriched with high loads of $\mathrm{As}, \mathrm{Cd}, \mathrm{Pb}$ and $\mathrm{Zn}, \mathrm{S}$. alba accumulated higher amounts of Cd and $\mathrm{Zn}$ in comparison to the results obtained in our study, while leaf uptake of As and Pb was similar (Vysloužilová et al. 2003).

PTEs in Juglans regia

Juglans regia accumulated $\mathrm{Cr}$ in its roots at levels considered toxic for plants at all sampling sites except VRH

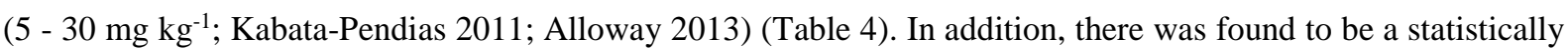


significant difference between VRH and the other sites in terms of the obtained content of $\mathrm{Cr}$ in $J$. regia roots (Table 4). Copper deficiency (2-5 $\mathrm{mg} \mathrm{kg}^{-1}$ ) was observed in its roots at MOJ, VRH and JAS and Zn deficiency (10-20 $\left.\mathrm{mg} \mathrm{kg}^{-1}\right)$ at VRH, CAT and JAS (Kabata-Pendias 2011) (Table 4).

In J. regia leaves, toxic levels of As were measured at LIT, VRH and CAT (5 - $20 \mathrm{mg} \mathrm{kg}^{-1}$; KabataPendias 2011; Alloway 2013), while a deficit of $\mathrm{Cu}$ in leaves (2 - $\left.5 \mathrm{mg} \mathrm{kg}^{-1}\right)$ was found at VRH, CAT, JAS and SRM, and of Zn (10 - $\left.20 \mathrm{mg} \mathrm{kg}^{-1}\right)$ at all sampling sites except LIT and RAD (Kabata-Pendias 2011) (Table 5). The LIT, VRH and CAT sampling sites stand out from the others in terms of the obtained content of As in the leaves of this species (Table 5). The deficiency of $\mathrm{Cu}$ and $\mathrm{Zn}$ observed in the roots and leaves of J. regia is probably due to their antagonism with As and Cr (Kabata-Pendias 2011), which were accumulated in a toxic range.

Compared to the results of the present study, Antonijević et al. (2012) measured similar As levels in roots near the flotation tailings pond of the copper mining and smelting complex in Bor, Serbia, but a slightly higher content in leaves (up to $8 \mathrm{mg} \mathrm{kg}^{-1}$ ). The latter is to be expected, bearing in mind that flotation tailings generally contain higher amounts of PTEs potentially available to plants (Antonijević et al. 2012). The available literature on Cr accumulation by J. regia mostly relates to its accumulation in branches, shells and kernels. In a study conducted by Arik and Yaldiz (2010) in an area with different types of ore deposits in western Turkey, Cr levels in J. regia branches were lower than those measured in the roots in our study. The same authors measured a lower As content in branches compared to the level in leaves in our research. Furthermore, numerous studies have shown that $\mathrm{Cr}$ levels in shells and kernels were lower than those obtained for J. regia leaves and roots in our study (Özcan 2008; Arpadjan et al. 2013; Tošić et al. 2014). From the current results, it is impossible to say with certainty whether the high $\mathrm{Cr}$ content measured in $J$. regia roots is species specific, demonstrating the need for further research. In terms of the accumulation of the other examined PTEs, similar levels of $\mathrm{Cu}$ and $\mathrm{Zn}$ were reported in a study by Sawidis et al. (2002) in J. regia leaves growing on deposits of lignite in Ptolemais, Greece. Moreover, Ghaderian and Ghotbi Ravandi (2012) reported similar Zn levels in J. regia leaves from the Sarcheshmeh copper mining area in Iran. In research conducted by Nečemer at al. (2008), Cd and Pb levels in the leaves of this species from a non-polluted site in Slovenia (Zaplana near Vrhnika) were determined using different methods, but the results revealed similar levels as in the present study.

\section{PTEs in Populus nigra}

Populus nigra accumulated various PTEs in its roots at levels considered toxic for plants: As (5 - $\left.20 \mathrm{mg} \mathrm{kg}^{-1}\right)$ at VRH and CAT and Cr (5 - $30 \mathrm{mg} \mathrm{kg}^{-1}$ ) at MOJ and SAB (Kabata-Pendias 2011; Alloway 2013). The ANOVA test revealed a significant difference between VRH and CAT and the other sites in terms of As content, as well as between SAB and the other sites when it came to $\mathrm{Cr}$ content in P. nigra leaves (Table 4). A deficit of $\mathrm{Cu}$ (2 - 5 $\mathrm{mg} \mathrm{kg}$ ) was measured at CAT and of Zn (10 - $\left.20 \mathrm{mg} \mathrm{kg}^{-1}\right)$ at MOJ and VRH (Kabata-Pendias 2011) (Table 4).

In $P$. nigra leaves, toxic levels were measured for Zn at CAT, ZAG and SAB (Table 5), while it was in the deficit range for plant tissues at SRM $\left(10.43 \mathrm{mg} \mathrm{kg}^{-1}\right)$. In addition, a copper deficit was measured at CAT (4.78 mg kg${ }^{-1}$ ), ZAG (4.36 mg kg$\left.{ }^{-1}\right)$, SRM (4.17 $\left.\mathrm{mg} \mathrm{kg}^{-1}\right)$ and BEO (4.06 mg kg-1) (Table 5). There is a 
statistically significant difference between the CAT, ZAG and SAB sites and the other locations in terms of Zn content in P. nigra leaves (Table 5).

At CAT, the deficit of $\mathrm{Cu}$ in roots is probably the result of the toxic levels of As due to the possible antagonistic relationship between these elements (Tang and Miller 1991). However, it could also be a result of the elevated levels of $\mathrm{Cr}$ in P. nigra roots ( $>0.5 \mathrm{mg} \mathrm{kg}^{-1}$; Kabata-Pendias 2011), bearing in mind the antagonistic relationship between $\mathrm{Cu}$ and $\mathrm{Cr}$ in plants. The $\mathrm{Cu}$ deficit in leaves at CAT and ZAG is most probably related to the toxic content of $\mathrm{Zn}$ because of the antagonistic relationship between these elements (Kabata-Pendias 2011). Plants most likely uptake these elements using the same mechanism and therefore one may inhibit the uptake of the other (Kabata-Pendias 2011). Copper levels are closely associated with soil texture and are usually the lowest in sandy soils (Kabata-Pendias and Mukhjeree 2007). Since the study by Pavlović et al. (2019) reported the texture of soils at CAT as being sandy loam, soil texture could also contribute to the lower uptake of $\mathrm{Cu}$ in the analysed plants.

At those sites where As content in roots was below the level of detection, Zn uptake was found to be at optimum levels for plant growth and development. Even so, at VRH and CAT, where As is in the toxic range, $P$. nigra accumulated lower amounts of $\mathrm{Zn}$, which may be a consequence of the antagonistic relationship between them (Kabata-Pendias 2011).

As with the genus Salix, species of the genus Populus have been shown to be good accumulators of $\mathrm{Cd}$ and Zn (Djingova et al. 1999; Sawidis et al. 2002; Baslar et al. 2005; Stobrawa and Lorenc-Plucińska 2007; Domínguez et al. 2008; Zacchini et al. 2011), which is in accordance with the results from this study. Namely, our results for $\mathrm{Zn}$ content in the leaves of $P$. nigra are similar to those obtained in research by Sawidis et al. (2002) on P. nigra leaves in an area with large coal-fired plants in Greece, as well as research conducted by Djingova et al. (1999) on the leaves of $P$. nigra 'Italica' growing near Cu smelter, metallurgical, cement and chemical plants. Meanwhile, Baslar et al. (2005) measured similar or lower $\mathrm{Zn}$ levels in P. nigra leaves in industrial (metallurgical, electronic, textile, and cement plants and a petroleum refinery), urban and suburban areas in Turkey.

On the other hand, our results point to elevated or toxic $\mathrm{Cr}$ content, as well as toxic As content in $P$. nigra roots at VRH and CAT. Similar Cr levels were reported in the leaves of this species growing in a copper mine area along the Nure river valley (Dinelli and Lombini 1996), as well as in the roots of this species, very often near the $\mathrm{Cu}$ smelter in Glogow, Poland (Stobrawa and Lorenc-Plucińska 2007). Moreover, similar As levels were reported in the leaves of $P$. nigra 'Italica', near metallurgical, chemical and cement plants in Bulgaria (Djingova et al. 1999), while Cr content was lower compared to our results.

When comparing the accumulation capacity of these three species, it was established that S. alba accumulated the highest content of PTEs in roots, except for $\mathrm{Cr}$ in the roots of J. regia. A similar accumulation pattern was observed for PTE levels in the leaves of the analysed plants. Salix alba accumulated the highest levels of $\mathrm{Cd}, \mathrm{Cu}, \mathrm{Ni}, \mathrm{Pb}$ and $\mathrm{Zn}$, while the leaves of $J$. regia accumulated the highest amounts of As and $\mathrm{Cr}$. Populus nigra has been shown to be the least effective for As accumulation in roots and $\mathrm{Cr}, \mathrm{Ni}$ and $\mathrm{Pb}$ accumulation in leaves. 
The phytostabilisation process reduces the mobility of PTEs, their leaching into ground water, and their bioavailability for entry into the food chain (Chojnacka et al. 2005), while through the phytoextraction process, plants transport and concentrate PTEs from the soil into their aerial parts (Yoon et al. 2006; Cui et al. 2007). The use of tolerant plant species to stabilise PTEs in soils could improve conditions for the natural attenuation or stabilisation of these pollutants. In order to evaluate the potential of the selected plants for the phytoextraction and phytostabilisation of PTEs, the bioaccumulation (BCF) and translocation (TF) factors were calculated (Table 6). If plants are able to retain PTEs in their roots, the TF has to be lower than 1, while the BCF can be either lower or higher than 1 indicating their potential for phytostabilisation. Plants can be regarded as phytoextractors if the TF is higher than 1, which indicates the transport of PTEs from roots to leaves (Fitz and Wenzel 2002). Leaves are considered to be the main sink for pollutants and therefore they are usually more sensitive to their effects than other plant parts (Bargagli 1998), which is important from the environmental standpoint due to leaffall which could further enrich soils with PTEs, especially in the case of $\mathrm{TF}>1$, and the existence of significant correlations of element levels in soils and leaves (Robinson et al. 2003).

In this study, highly significant correlations were found between As, Ni and Zn levels in soils and leaves (Table 7). Although the correlation between As content in soils and the leaves of $J$. regia was not statistically significant (ns), the high TF and toxic level of As in leaves could contribute to soil enrichment with this element in the future.

Table 6 Bioconcentration (BCFs) and translocation (TFs) factors for PTEs in plants at selected sampling sites

\begin{tabular}{|c|c|c|c|c|c|c|c|c|c|c|c|c|c|c|c|}
\hline \multicolumn{2}{|c|}{ Elements } & \multicolumn{7}{|c|}{ BCFs } & \multicolumn{7}{|c|}{ TFs } \\
\hline tes & & As & Cd & $\mathrm{Cr}$ & $\mathrm{Cu}$ & $\mathbf{N i}$ & $\mathbf{P b}$ & $\mathbf{Z n}$ & As & Cd & $\mathrm{Cr}$ & $\mathrm{Cu}$ & $\mathbf{N i}$ & $\mathbf{P b}$ & Zn \\
\hline \multirow{12}{*}{ 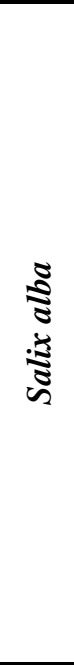 } & MOJ & 0.56 & 1 & I & 0.16 & 1 & 0.04 & 0.35 & 1.49 & 2.00 & 1 & 2.16 & 1 & 1 & 4.04 \\
\hline & RAD & 0.69 & I & I & 0.13 & I & 0.09 & 0.31 & I & 1.00 & I & 1.92 & I & I & 8.56 \\
\hline & LIT & 0.50 & I & I & 0.41 & 0.15 & 0.13 & 0.43 & I & 1.00 & I & 1.22 & 0.23 & 0.23 & 2.19 \\
\hline & VRH & 0.30 & I & 0.02 & 0.37 & 0.02 & 0.11 & 0.49 & I & 6.20 & I & 0.68 & I & 1 & 6.87 \\
\hline & CAT & 0.38 & I & 0.03 & 0.02 & 0.03 & 0.07 & 0.55 & l & 11.66 & l & 0.97 & I & I & 5.80 \\
\hline & ZAG & I & I & 0.08 & 0.64 & 0.06 & 0.21 & 1.18 & I & 2.52 & I & 0.73 & I & I & 1.71 \\
\hline & JAS & 0.59 & I & 0.02 & 0.43 & 0.03 & 0.11 & 0.52 & I & 0.50 & I & 1.51 & 1.29 & I & 3.84 \\
\hline & SLB & 0.54 & I & 0.06 & 0.50 & 0.09 & 0.23 & 0.77 & I & 0.99 & 0.92 & 0.88 & 0.53 & 0.35 & 3.72 \\
\hline & ZUP & 0.53 & I & 0.12 & 0.48 & 0.19 & 0.29 & 0.55 & 0.23 & 0.54 & 0.18 & 0.55 & 0.22 & 0.14 & 2.18 \\
\hline & SRM & 0.40 & 11.08 & 0.06 & 0.53 & 0.13 & 0.18 & 0.50 & I & 0.50 & I & 0.35 & I & I & 3.09 \\
\hline & SAB & 0.39 & 32.00 & 0.06 & 0.52 & 0.09 & 0.22 & 0.79 & 0.98 & 0.30 & 1.58 & 0.51 & 1.30 & 0.84 & 1.63 \\
\hline & BEO & 0.2 & 120.78 & 6 & 0.45 & 0.08 & 4 & 1.25 & 1 & 0.01 & I & 0.39 & 0.29 & I & 0.46 \\
\hline \multirow{6}{*}{$\begin{array}{l}\stackrel{\Xi}{\Xi} \\
\frac{9}{5} \\
\frac{5}{5}\end{array}$} & MOJ & 0.81 & 1 & 0.39 & 0.32 & 0.03 & 0.10 & 0.25 & 0.40 & r & 0.23 & 1.37 & 1 & 0.22 & 0.75 \\
\hline & RAD & 0.4 & I & 0.23 & 0.39 & 0.06 & 0.06 & 0.43 & 0.8 & 1 & 0.32 & 1.17 & 0.39 & 0.69 & 0.90 \\
\hline & LIT & 0.5 & I & 0.31 & 0.63 & 0.06 & 0.05 & 0.58 & 2.12 & I & 0.20 & 0.65 & 0.57 & 1 & 0.92 \\
\hline & VRH & 0.44 & I & 0.19 & 0.10 & I & 0.07 & 0.02 & 2.92 & I & 0.22 & 2.26 & I & I & 1.00 \\
\hline & CAT & 0.45 & I & 0.77 & 0.02 & 0.19 & 0.18 & 0.19 & 1.69 & I & 0.03 & 0.48 & I & I & 0.44 \\
\hline & JAS & 1.12 & 1 & 0.52 & 0.29 & 0.16 & 0.23 & 0.16 & 0.31 & 1 & 0.38 & 1.35 & 0.42 & 0.50 & 1.01 \\
\hline
\end{tabular}




\begin{tabular}{cccccccccccccccc} 
SRM & 0.12 & 3.75 & 0.12 & 0.31 & 0.09 & 0.12 & 0.20 & $\mathbf{1 . 3 0}$ & $/$ & 0.14 & 0.43 & 0.20 & 0.31 & 0.28 \\
\hline MOJ & 0.58 & $/$ & 0.34 & 0.71 & 0.34 & 0.07 & 0.19 & $/$ & $/$ & $/$ & 0.54 & $/$ & 0.18 & $\mathbf{2 . 1 8}$ \\
& VRH & $\mathbf{1 . 2 1}$ & $/$ & 0.16 & 0.29 & 0.05 & 0.08 & 0.16 & $/$ & $/$ & 0.05 & $\mathbf{1 . 1 6}$ & $/$ & 0.18 & $\mathbf{2 . 1 8}$ \\
& CAT & 0.71 & $/$ & 0.12 & 0.01 & 0.04 & 0.11 & 0.26 & $/$ & $\mathbf{6 . 8 7}$ & 0.06 & $\mathbf{1 . 1 9}$ & 0.27 & $\mathbf{1 . 0 4}$ & $\mathbf{5 . 2 9}$ \\
$\mathbf{\Xi}$ & ZAG & $/$ & $/$ & 0.14 & 0.62 & 0.10 & 0.11 & $\mathbf{1 . 2 0}$ & $/$ & $\mathbf{1 . 8 7}$ & $/$ & 0.62 & $/$ & 0.24 & $\mathbf{2 . 4 6}$ \\
$\stackrel{\Xi}{\Xi}$ & JAS & $/$ & $/$ & 0.17 & 0.43 & 0.11 & 0.11 & $\mathbf{1 . 2 0}$ & $/$ & $\mathbf{1 . 4 9}$ & 0.08 & $\mathbf{1 . 3 4}$ & 0.18 & 0.21 & $\mathbf{1 . 2 2}$ \\
$\mathbf{\Xi}$ & ZUP & $/$ & $/$ & 0.02 & 0.28 & 0.05 & 0.05 & 0.31 & $/$ & $\mathbf{3 . 0 0}$ & $/$ & 0.74 & 0.43 & $/$ & $\mathbf{3 . 4 8}$ \\
$\mathbf{\Xi}$ & SRM & $/$ & $\mathbf{4 . 3 3}$ & 0.05 & 0.23 & 0.08 & 0.09 & 0.23 & $/$ & $\mathbf{1 . 0 0}$ & $/$ & 0.56 & 0.03 & $/$ & 0.40 \\
& SAB & $/$ & $\mathbf{7 . 3 6}$ & 0.06 & 0.23 & 0.07 & 0.10 & 0.33 & $/$ & $\mathbf{1 . 5 0}$ & $/$ & 0.62 & 0.06 & $/$ & $\mathbf{3 . 9 1}$ \\
BEO & $/$ & 0.72 & 0.03 & 0.14 & 0.05 & 0.03 & 0.38 & $/$ & $\mathbf{1 . 4 8}$ & $/$ & 0.48 & $/$ & $/$ & 0.59 \\
\hline
\end{tabular}

Table 7 Correlations of PTE levels in the roots and leaves of the examined species with the soils in which they grow

\begin{tabular}{|c|c|c|c|c|c|c|c|}
\hline & & & & Soil & & & \\
\hline Plants & As & Cd & $\mathrm{Cr}$ & $\mathrm{Cu}$ & $\mathbf{N i}$ & $\mathbf{P b}$ & Zn \\
\hline \multicolumn{8}{|l|}{ Roots } \\
\hline Salix alba & $0.764^{* * *}$ & $0.752^{* * *}$ & $\mathbf{0 . 8 1 1}^{* * *}$ & $0.570^{* * *}$ & $0.732^{* * *}$ & $0.493^{* * *}$ & $0.682^{* * *}$ \\
\hline Juglans regia & $-0.348^{*}$ & $0.615^{* * *}$ & $0.571^{* * *}$ & $0.434^{* *}$ & $0.627^{* * *}$ & $0.658^{* * *}$ & 0.296 \\
\hline Populus nigra & $-0.519^{* * *}$ & $0.443^{* * *}$ & 0.162 & 0.041 & $0.654^{* * *}$ & $0.684^{* * *}$ & 0.148 \\
\hline \multicolumn{8}{|l|}{ Leaves } \\
\hline Salix alba & 0.108 & 0.083 & $0.498^{* * *}$ & $0.399^{* * *}$ & $0.517^{* * *}$ & -0.026 & $0.532^{* * *}$ \\
\hline Juglans regia & 0.247 & I & -0.135 & $-0.451^{* *}$ & $0.407^{* *}$ & 0.154 & -0.240 \\
\hline Populus nigra & $0.590^{* * *}$ & 0.018 & $-0.276^{*}$ & $-0.373^{* *}$ & $0.525^{* * *}$ & $-0.307^{*}$ & -0.082 \\
\hline
\end{tabular}

The results showed that despite significant positive correlations between the levels of all the examined elements in soil and roots, the BCF for $S$. alba was less than $1(\mathrm{BCF}<1)$ at all the sampling sites for all the elements except Cd at SRM (11.08), SAB (32.00) and BEO (120.78) and Zn at ZAG (1.18) and BEO (1.25). Similarly, significant positive correlations existed between PTE levels in the roots and leaves of $S$. alba, with a TF for Cd higher than 1 recorded at MOJ, RAD, LIT, VRH, CAT and ZAG, while the values for Zn were also higher than 1 at all sampling sites except BEO (Table 6). In addition to Cd and Zn, S. alba also transferred Cu to leaves at MOJ, RAD, LIT and JAS, Ni at JAS and SAB, Cr at SAB, and As at MOJ (Table 6).

The results indicate the potential of $S$. alba for the phytoextraction (in the upper stretch of the river) and phytostabilisation (in the middle and lower stretches) of $\mathrm{Cd}$ and $\mathrm{Cu}$. Furthermore, its potential for the phytoextraction of Zn was shown at all sampling sites except BEO. Previous research on Salix sp., especially on Salix viminalis and Salix dasyclados, has shown that these species have good $\mathrm{Cd}$ and $\mathrm{Zn}$ accumulation capacity both in leaves (Punshon and Dickinson 1997; Meers et al. 2003, 2007) and roots (Stobrawa and LorencPlucińska 2007; Zacchini et al. 2011). Additionally, the results of our research indicate that S. alba possesses the capacity for the phytostabilisation of $\mathrm{Pb}$ (at all sampling sites), and $\mathrm{Cr}$ and $\mathrm{Ni}$ (at all sampling sites except $\mathrm{SAB}$ ). Vandecasteele et al. (2005) had similar results in their research on Salix sp. clones. 
Significant correlations were established between the levels of all the examined elements in soil and the roots of $J$. regia, except As $\left(-0.348^{*}\right)$ and Zn (ns) at all the sampling sites. However, the BCF was less than 1 for all the examined elements except As at JAS (1.12) and Cd at SRM (3.75) (Table 6). Even though significant positive correlations were also established between roots and leaves for $\mathrm{Zn}$ (0.742), Ni (0.608) and $\mathrm{Pb}(0.403)$ (Table 8), only As at LIT, VRH, CAT and SRM, Cu at MOJ, RAD, VRH and JAS, and Zn at VRH and JAS was efficiently transported to leaves (TF $>1$ ) (Table 6)

The results indicate the potential of this species for the phytoextraction of As, as well as for the phytostabilisation of Cr, Ni and Pb. Similarly, Saqib et al. (2013) and Ozen and Yaman (2016) in their research demonstrated the uptake capacity of J. regia for As and Pb in its leaves, while Marmiroli et al. $(1999,2005)$ showed that this species accumulates $\mathrm{Cr}$ and $\mathrm{Pb}$ in its roots. However, Antonijević et al. (2012) previously reported high BCFs and TFs for Zn, which does not coincide with the results from our study, given the fact that Zn content in roots and leaves was low, and even deficient at most of the sampling sites.

For P. nigra significant correlations were established between the levels of Cd (0.443), Ni (0.654) and $\mathrm{Pb}(0.684)$ in soil and roots, while for As, there was a significant negative correlation (-0.519**) (Table 7). However, as with S. alba, the BCF for P. nigra was higher than 1 for Cd at SRM (4.33) and SAB (7.36), for Zn at ZAG and JAS (1.25), and for As at VRH (1.21) (Table 6). In addition, significant positive correlations were established for $\mathrm{Cd}\left(0.348^{*}\right)$, Ni $(0.345)$ and $\mathrm{Zn}\left(0.413^{*}\right)$ in its roots and leaves (Table 8), with $P$. nigra efficiently transporting $\mathrm{Cd}$ at all sampling sites, $\mathrm{Zn}$ at all sites except SRM and BEO, Cu at VRH (1.16), CAT (1.19) and JAS (1.34) and Pb at CAT (1.04) from its roots to its leaves (Table 6).

The results obtained during our research indicate the potential of $P$. nigra for the phytoextraction of Cd and $\mathrm{Zn}$. This species was also proven to be good for the phytostabilisation of $\mathrm{Cr}, \mathrm{Ni}$ and $\mathrm{Pb}$, and to some extent Cu. Similarly, in their research Jakovljević et al. (2014) showed that $P$. nigra also possesses potential for Cd phytoextraction, while Baldantoni et al. (2014) found high TFs for $\mathrm{Cd}$ and $\mathrm{Zn}$, and the potential for $\mathrm{Pb}$ phytostabilisation in $P$. nigra clones.

The S. alba and P. nigra species demonstrated that they possess the potential for the phytoextraction of $\mathrm{Cd}$ and $\mathrm{Zn}$, while J. regia has the potential for the phytoextraction of As. On the other hand, S. alba shows potential for the phytostabilisation of $\mathrm{Cd}$ and $\mathrm{Cu}$ in the middle and lower reaches of the river. J. regia is a phytostabiliser for As, $\mathrm{Cr}, \mathrm{Ni}$ and $\mathrm{Pb}$. P. nigra also proved to be good for the phytostabilisation of $\mathrm{Cr}, \mathrm{Ni}$ and $\mathrm{Pb}$, and to some extent $\mathrm{Cu}$. The results also showed similar variations in the BCFs and TFs for $\mathrm{Cu}$ in all the examined species, which can be linked to the physiological mechanisms of uptake and accumulation of $\mathrm{Cu}$ as an essential element (Domínguez et al. 2008).

Table 8 Correlations of PTE levels in the roots and leaves of the examined species

\begin{tabular}{cccccccc}
\hline \multirow{2}{*}{ Roots } & \multicolumn{7}{c}{ Salix alba } \\
\cline { 2 - 8 } Leaves & \multicolumn{1}{c}{ As } & Cd & Cr & Cu & Ni & Pb & Zn \\
\hline As & $\mathbf{0 . 2 9 9}^{*}$ & 0.139 & $\mathbf{0 . 2 4 8}^{*}$ & 0.119 & $\mathbf{0 . 2 3 4}^{*}$ & $\mathbf{0 . 3 2 5}^{* *}$ & $\mathbf{0 . 2 6 9}^{*}$ \\
Cd & 0.089 & $\mathbf{0 . 3 3 9}^{* *}$ & $\mathbf{0 . 4 2 9}^{* * *}$ & $\mathbf{0 . 4 0 7}^{* * *}$ & $\mathbf{0 . 2 7 6}^{*}$ & 0.081 & $\mathbf{0 . 5 0 8}^{* * *}$ \\
Cr & $\mathbf{0 . 4 6 1}^{* * *}$ & $\mathbf{0 . 3 4 8}^{* *}$ & $\mathbf{0 . 4 2 0}^{* * *}$ & $\mathbf{0 . 3 3 0}^{* *}$ & $\mathbf{0 . 6 1 9}^{* * *}$ & $\mathbf{0 . 5 3 5}^{* * *}$ & 0.087 \\
Cu & $\mathbf{0 . 7 3 7}^{* * *}$ & $\mathbf{0 . 8 1 5}^{* * *}$ & $\mathbf{0 . 7 1 1}^{* * *}$ & $\mathbf{0 . 7 2 1}^{* * *}$ & $\mathbf{0 . 7 7 3}^{* * *}$ & $\mathbf{0 . 5 6 9}^{* * *}$ & $\mathbf{0 . 5 2 4}^{* * *}$ \\
Ni & $\mathbf{0 . 5 7 1}^{* * *}$ & $\mathbf{0 . 5 2 8}^{* * *}$ & $\mathbf{0 . 5 1 5}^{* * *}$ & $\mathbf{0 . 4 3 1}^{* * *}$ & $\mathbf{0 . 5 7 0}^{* * *}$ & $\mathbf{0 . 6 0 6}^{* * *}$ & $\mathbf{0 . 2 9 9}^{*}$
\end{tabular}




\begin{tabular}{|c|c|c|c|c|c|c|c|}
\hline $\mathbf{P b}$ & $0.452^{* * *}$ & $0.336^{* *}$ & $0.401^{* * *}$ & $0.317^{* *}$ & $0.606^{* * *}$ & $0.531^{* * *}$ & 0.078 \\
\hline $\mathbf{Z n}$ & $0.234^{*}$ & 0.189 & 0.071 & 0.119 & -0.091 & -0.043 & $0.288^{*}$ \\
\hline \multicolumn{8}{|c|}{ Juglans regia } \\
\hline & As & Cd & $\mathrm{Cr}$ & $\mathrm{Cu}$ & $\mathbf{N i}$ & $\mathbf{P b}$ & Zn \\
\hline$\overline{\text { As }}$ & -0.154 & -0.183 & -0.178 & -0.183 & -0.226 & $-0.365^{*}$ & -0.299 \\
\hline Cd & l & l & / & l & l & l & l \\
\hline $\mathrm{Cr}$ & 0.109 & 0.086 & 0.101 & 0.131 & 0.125 & -0.120 & 0.103 \\
\hline $\mathbf{C u}$ & -0.001 & $-0.599^{* * *}$ & $-0.358^{*}$ & 0.198 & $-0.355^{*}$ & -0.179 & $0.312^{*}$ \\
\hline $\mathbf{N i}$ & -0.129 & $0.520^{* * *}$ & $0.357^{*}$ & $0.579^{* * *}$ & $0.608^{* * *}$ & -0.188 & $0.450^{* *}$ \\
\hline $\mathbf{P b}$ & 0.206 & 0.154 & 0.175 & 0.084 & 0.190 & $0.403^{* *}$ & 0.151 \\
\hline $\mathbf{Z n}$ & -0.136 & $-0.366^{*}$ & -0.104 & $0.644^{* * *}$ & -0.010 & -0.213 & $0.742^{* * *}$ \\
\hline \multicolumn{8}{|c|}{ Populus nigra } \\
\hline & As & Cd & $\mathrm{Cr}$ & $\mathrm{Cu}$ & $\mathbf{N i}$ & $\mathbf{P b}$ & $\mathbf{Z n}$ \\
\hline As & $-0.694^{* * *}$ & $0.324^{*}$ & 0.060 & 0.177 & $0.652^{* * *}$ & -0.124 & $0.606^{* * *}$ \\
\hline Cd & -0.218 & $0.348^{* *}$ & -0.229 & -0.240 & -0.030 & 0.038 & $0.416^{* *}$ \\
\hline $\mathrm{Cr}$ & $0.478^{* * *}$ & -0.239 & -0.080 & $-0.826^{* * *}$ & $-0.640^{* * *}$ & -0.225 & -0.119 \\
\hline $\mathbf{C u}$ & 0.217 & -0.080 & 0.075 & -0.191 & -0.248 & -0.214 & -0.218 \\
\hline $\mathbf{N i}$ & $-0.310^{*}$ & 0.097 & 0.076 & -0.001 & $0.345^{*}$ & 0.034 & 0.173 \\
\hline $\mathbf{P b}$ & $0.656^{* * *}$ & $-0.339^{*}$ & -0.118 & $-0.465^{* * *}$ & $-0.779^{* * *}$ & 0.042 & $-0.368^{* *}$ \\
\hline Zn & -0.152 & $0.424^{* *}$ & -0.162 & -0.045 & -0.042 & 0.100 & $0.413^{* *}$ \\
\hline
\end{tabular}

Differences in PTE accumulation capacity between the examined species

In order to highlight the variations between PTE accumulation in S. alba, J. regia and P. nigra and discover patterns, canonical discriminant analysis (CDA) was performed separately for element content in the species' roots and leaves (Fig 2 and 3; Table 9).

Table 9 Standardised Coefficients for Canonical Variables

\begin{tabular}{crrrr}
\hline & \multicolumn{2}{c}{ CDA roots } & \multicolumn{2}{c}{ CDA leaves } \\
\hline & DC 1 & DC 2 & \multicolumn{1}{c}{ DC 1 } & \multicolumn{1}{c}{ DC 2 } \\
\hline As & 0.085 & $\mathbf{- 0 . 8 9 9}$ & -0.315 & -0.126 \\
Cd & 0.153 & -0.292 & 0.239 & 0.195 \\
Cr & $\mathbf{- 0 . 5 0 8}$ & -0.299 & -0.042 & -0.404 \\
$\mathbf{C u}$ & 0.245 & -0.291 & 0.191 & -0.325 \\
$\mathbf{~ N i}$ & 0.167 & -0.077 & 0.127 & -0.245 \\
$\mathbf{P b}$ & 0.148 & $\mathbf{- 0 . 5 9 9}$ & 0.029 & -0.116 \\
$\mathbf{Z n}$ & 0.281 & -0.216 & $\mathbf{0 . 5 4 1}$ & -0.171 \\
\hline Eigenval & 1.597 & 0.261 & 3.274 & 0.621 \\
Cum.Prop & 0.859 & 1.000 & 0.840 & 1.000 \\
\hline
\end{tabular}

Values below -0.5 and above 0.5 are denoted in bold 


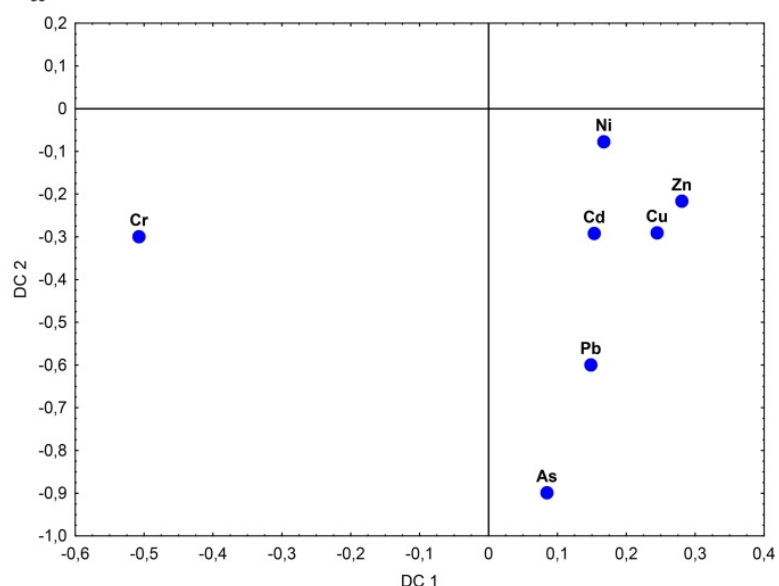

b

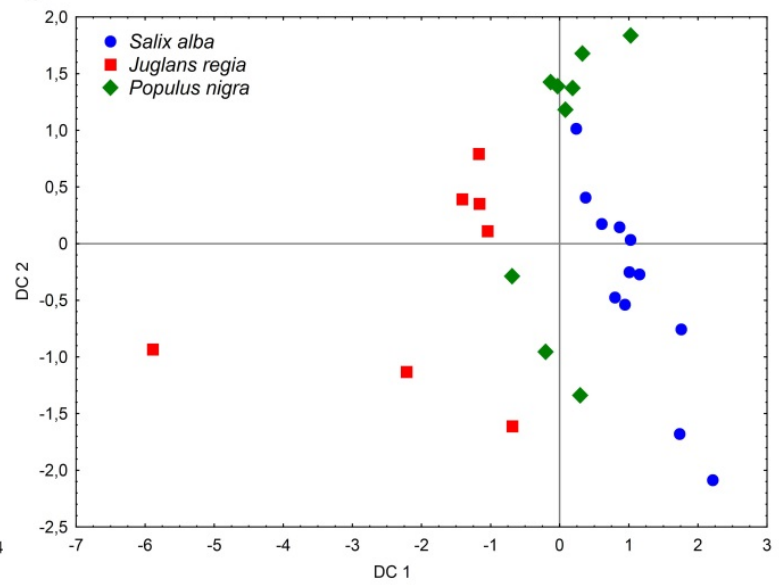

Fig. 2 Differences in the accumulation patterns of the examined species in roots a) elements b) species
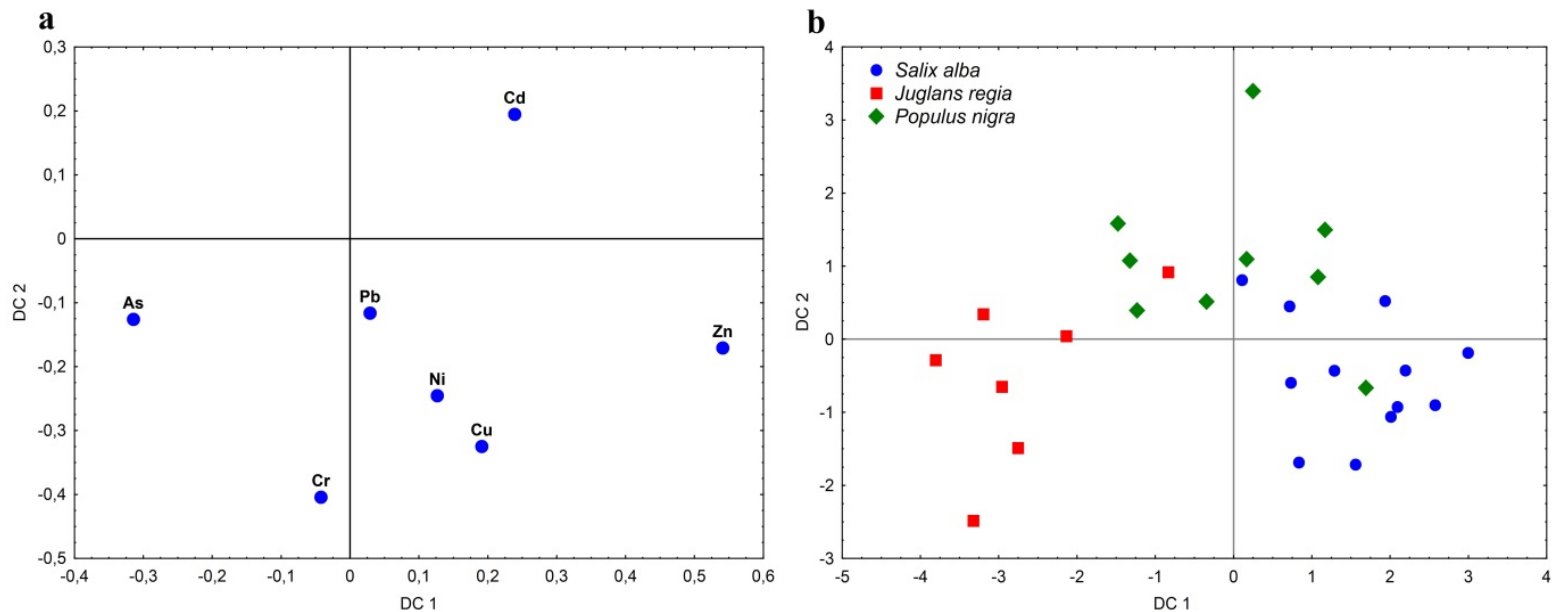

Fig. 3 Differences in the accumulation patterns of the examined species in leaves a) elements b) species

Based on the first component (DC 1), which explains $85.9 \%$ of variance, and the second component (DC 2), which explains $14.1 \%$ of differences, J. regia roots are separated from the other two species, probably due to higher $\mathrm{Cr}$ accumulation. The separation between $S$. alba and P. nigra is mainly impacted by the higher levels of $\mathrm{As}, \mathrm{Pb}, \mathrm{Cd}, \mathrm{Cu}, \mathrm{Ni}$ and $\mathrm{Zn}$ in the roots of the former. In leaves, the first component (DC 1) explained $84 \%$ of variance and separates S. alba from the other two species due to a higher leaf uptake of $\mathrm{Zn}, \mathrm{Cu}, \mathrm{Ni}$ and $\mathrm{Pb}$, as well as J. regia due to a higher uptake of As and Cr. The second component (DC 2), which explains $16 \%$ of variance, separates the leaves of $P$. nigra from the other examined species due to Cd accumulation (Fig. 3).

\section{Conclusion}

The present study focused on the potential of Salix alba, Juglans regia and Populus nigra to accumulate PTEs (As, Cd, Cr, Cu, Ni, Pb and $\mathrm{Zn}$ ) in the riparian zone of the River Sava. Their levels were determined in soils at 
selected sampling sites with specific anthropogenic sources of pollution, as well as in the roots and leaves of the selected species.

Despite the fact that levels above the background values for European soils were measured for $\mathrm{Cr}, \mathrm{Cu}$ and $\mathrm{Ni}$ in some soils of the River Sava's riparian zone, with moderate ( $\mathrm{Zn})$, significant $(\mathrm{Pb})$ and even very high $(\mathrm{Cu})$ enrichment, the uptake, accumulation and transfer of the elements in the aboveground parts of the examined species differed from species to species. The results obtained in the present study revealed close similarity in the accumulation of PTEs in the leaves of S. alba and P. nigra. Both species predominantly showed phytoextraction potential for $\mathrm{Cd}$ and $\mathrm{Zn}$ in the examined soils, with $S$. alba being slightly more efficient than $P$. nigra. However, these two species differ in their phytostabilisation potential, given the fact that $S$. alba demonstrated potential for $\mathrm{Cd}$ and $\mathrm{Cu}$ phytostabilisation, while $\mathrm{P}$. nigra revealed potential for the phytostabilisation of $\mathrm{Cr}, \mathrm{Ni}$ and $\mathrm{Pb}$, and to some extent $\mathrm{Cu}$. On the other hand, J. regia showed potential for the phytoextraction of As and the phytostabilisation of As, $\mathrm{Cr}, \mathrm{Ni}$ and $\mathrm{Pb}$. The phytostabilisation and phytoextraction potential of the species was additionally confirmed through the BCFs and TFs, correlations and CDA analyses. Through comparing the accumulation capacity of these three species, it was established that $S$. alba accumulated the highest levels of PTEs in roots, except for $\mathrm{Cr}$ in the roots of $J$. regia. A similar accumulation pattern was observed in terms of PTE levels in the leaves of the analysed plants. Salix alba accumulated the highest amounts of $\mathrm{Cd}, \mathrm{Cu}, \mathrm{Ni}, \mathrm{Pb}$ and $\mathrm{Zn}$, while the leaves of $J$. regia accumulated the highest levels of As and Cr. Populus nigra was shown to be the least effective in terms of the accumulation of As in roots, and the accumulation of $\mathrm{Cr}, \mathrm{Ni}$ and $\mathrm{Pb}$ in leaves.

The positive correlations of element levels in soils and leaves indicated the possibility of the additional enrichment of soils with PTEs (especially As, Ni and Zn) due to leaf-fall, which can be a focus of future research.

\section{Acknowledgments}

This work has been supported by the European Communities 7th Framework Programme Funding under Grant agreement no. 603629-ENV-2013-6.2.1-Globaqua. The preparation of this manuscript was supported by the Ministry of Education, Science and Technological Development of the Republic of Serbia, grant no. 173018.

\section{Author's declaration}

This article is original and has not been previously published, in whole or in part. This work is not under consideration by any other journal elsewhere and its publication is approved by all authors. In case of the acceptance of this paper, it will not be published elsewhere in the same form, in English or in any other language, including electronically without the written consent of the copyright-holder.

\section{Conflict of interest}

The authors declare that they have no conflict of interest.

\section{References}

Adriano, D.C. (2001). Trace elements in terrestrial environments. Biogeochemistry, bioavailability and risk of metals. Springer, New York. ISBN 978-0-387-21510-5 
Alloway, B.J. (2013). Heavy metals in soils. Trace metals and metaloids in soils and their bioavailability. Environmental pollution (22), third edition. Springer, New York. ISBN 978-94-007-4470-7

Antonijević, M.M., Dimitrijević, M.D., Milić, S.M., Nujkić, M.M. (2012). Metal concentrations in the soils and native plants surrounding the old flotation tailings pond of the Copper Mining and Smelting Complex Bor (Serbia). Journal of Environmental Monitoring, 14(3), 866-877. https://doi.org/10.1039/c2em10803h

Arik, F., Yaldiz, T. (2010). Heavy metal determination and pollution of the soil and plants of southeast Tavşanli (Kütahya, Turkey). Clean (Weinh), 38(11), 1017-1030. https://doi.org/10.1002/clen.201000131

Arpadjan, S., Momchilova, S., Elenkova, D., Blagoeva, E. (2013). Essential and toxic microelement profile of walnut (Juglans regia L.) cultivars grown in industrially contaminated area - Evaluation for human nutrition and health. Journal of Food and Nutrition Research, 52(2), 121-127. ISSN: 1336-8672

Bai, J., Zhao, Q., Lu, Q., Wang, J., Reddy, K.R. (2015). Effects of freshwater input on trace element pollution in salt marsh soils of a typical coastal estuary China. Journal of Hydrology, 520, 186-192. https://doi.org/10.1016/j.jhydrol.2014.11.007

Baldantoni, D., Cicatelli, A., Bellino, A., Castiglione, S. (2014). Different behaviours in phytoremediation capacity of two heavy metal tolerant poplar clones in relation to iron and other trace elements. Journal of Environmental Management 146, 94-99. https://doi.org/10.1016/j.jenvman.2014.07.045

Barbieri, M. (2016). The Importance of Enrichment Factor (EF) and Geoaccumulation Index (Igeo) to Evaluate the Soil Contamination. Journal of Geology and Geophysics, 5(1), 1-4. https://doi.org/10.4172/23818719.1000237

Bargagli, R. (1998). Trace Elements in Terrestrial Plants: An Ecophysiological Approach to Biomonitoring and Biorecovery. Springer-Verlag, Berlin. ISBN 9783540645511

Baslar, S., Dogan, Y., Yenil, N., Karagoz, S., Bag, H. (2005). Trace element biomonitoring by leaves of Populus nigra L. from Western Anatolia, Turkey. Journal of Environmental Biology, 26(4), 665-668. ISSN: 0254-8704

Bedell, J.P., Capilla, X., Giry, C., Schwartz, C., Morel, J.L., Perrodin, Y. (2009). Distribution, movement and availability of $\mathrm{Cd}$ and $\mathrm{Zn}$ in a dredged sediment cultivated with Salix alba. Environmental and Experimental Botany, 67(2), 403-414. https://doi.org/10.1016/j.envexpbot.2009.08.001

Berlizov, A.N., Blum, O.B., Filby, R.H., Malyuk, I.A., Tryshyn, V.V. (2007) Testing applicability of black poplar (Populus nigra L.) bark to heavy metal air pollution monitoring in urban and industrial regions. Science of the Total Environment, 372, 693-706. https://doi.org/10.1016/j.scitotenv.2006.10.029

Bhargava, A., Carmona, F.F., Bhargava, M., Srivastava, S. (2012). Approaches for enhanced phytoextraction of heavy metals. Journal of Environmental Management, 105, 103-120. https://doi.org/10.1016/j.jenvman.2012.04.002

Chen, L., Gao, S., Zhu, P., Liu, Y., Hu, T., Zhang, J. (2014). Comparative study of metal resistance and accumulation of lead and zinc in two poplars. Plant Physiology, 151, 390-405. https://doi.org/10.1111/ppl.12120

Chojnacka, K., Chojnacki, A., Górecka, H., Górecki, H. (2005). Bioavailability of heavy metals from polluted soils to plants. Science of the Total Environment, 337(1-3), 175-182. https://doi.org/10.1016/j.scitotenv.2004.06.009

Cui, S., Zhou, Q.X., Chao, L. (2007). Potential hyperaccumulation of Pb, Zn, Cu and Cd in endurant plants distributed in an old smeltery, northeast China. Environmental Geology, 51, 1043-1048. https://doi.org/10.1007/s00254-006-0373-3

Čakmak, D., Perović, V., Antić-Mladenović, S., Kresović, M., Saljnikov, E., Mitrović, M., Pavlović, P. (2018). Contamination, risk, and source apportionment of potentially toxic microelements in river sediments and soil after extreme flooding in the Kolubara River catchment in Western Serbia. Journal of Soils and Sediments, 18, 1981-1993. https://doi.org/10.1007/s11368-017-1904-0

Dinelli, E., Lombini, A. (1996). Metal distributions in plants growing on copper mine spoils in Northern Apennines, Italy: The evaluation of seasonal variations. Applied Geochemistry, 11(1-2), 375-385. https://doi.org/10.1016/0883-2927(95)00071-2

Djingova, R., Wagner, G., Kuleff, I. (1999). Screening of heavy metal pollution in Bulgaria using Populus nigra "Italica". Science of the Total Environment, 234(1-3), 175-184. https://doi.org/10.1016/S00489697(99)00257-0

Domínguez, M.T., Marañón, T., Murillo, J.M., Schulin, R., Robinson, B.H. (2008). Trace element accumulation in woody plants of the Guadiamar Valley, SW Spain: A large-scale phytomanagement case study. Environmental Pollution, 152(1), 50-59. https://doi.org/10.1016/j.envpol.2007.05.021

Dos Santos Utmazian, M.N., Wieshammer, G., Vega, R., Wenzel, W.W. (2007). Hydroponic screening for metal resistance and accumulation of cadmium and zinc in twenty clones of willows and poplars. Environmental Pollution, 148(1), 155-165. https://doi.org/10.1016/j.envpol.2006.10.045 
Dragun, Z., Filipović Marijić, V., Vuković, M., Raspor, B. (2015). Metal Bioavailability in the Sava River Water. In: R. Milačič, J. Ščančar, M. Paunović (Vol Eds), The Sava River (pp. 123-155). Springer, Dodrecht, Heidelberg, New York, London ISBN 978-3-662-44034-6.

Du Laing, G., Rinklebe, J., Vandecasteele, B., Meers, E., Tack, F.M.G. (2009). Trace metal behaviour in estuarine and riverine floodplain soils and sediments: A review. Science of the Total Environment, 407(13), 3972-3985. https://doi.org/10.1016/j.scitotenv.2008.07.025

Fan, J., He, Z., Ma, L.Q., Stoffella, P.J. (2011). Accumulation and availability of copper in citrus grove soils as affected by fungicide application. Journal of Soils and Sediments, 11(4), 639-648. https://doi.org/10.1007/s11368-011-0349-0

Feizi, M., Jalali, M. (2015). Removal of heavy metals from aqueous solutions using sunflower, potato, canola and walnut shell residues. Journal of the Taiwan Institute of Chemical Engineers, 54, 125-136. https://doi.org/10.1016/j.jtice.2015.03.027

Fitz, W.J., Wenzel, W.W. (2002). Arsenic transformations in the soil-rhizosphere-plant system: fundamentals and potential application to phytoremediation. Journal of Biotechnology, 99, 259-278. https://doi.org/10.1016/S0168-1656(02)00218-3

Gaudet, M., Pietrini, F., Beritognolo, I., Iori, V., Zacchini, M., Massacci, A., Mugnozza, G.S., Sabatti, M. (2011). Intraspecific variation of physiological and molecular response to cadmium stress in Populus nigra L. Tree Physiology, 31, 1309-1318. https://doi.org/10.1093/treephys/tpr088

Gawlik, B.W., Bidoglio, G. (2006). Background values in European soils and sewage sludges PART III, Conclusions, comments and recommendations. European Commission, Directorate-General Joint Research Centre, Institute for Environment and Sustainability (Directive 86/278/EEC). https://publications.europa.eu/en/publication-detail/-/publication/f7081979-a8dc-4c2d-a7eb63b4dfdd9b83/language-en. Accessed 16 March 2019.

Ghaderian, S.M., Ghotbi Ravandi, A.A. (2012). Accumulation of copper and other heavy metals by plants growing on Sarcheshmeh copper mining area, Iran. Journal of Geochemical Exploration, 123, $25-32$. https://doi.org/10.1016/j.gexplo.2012.06.022

Gjorgieva, D., Kadifkova-Panovska, T., Bačeva, K., Stafilov, T. (2011). Assessment of heavy metal pollution in Republic of Macedonia using a plant assay. Archives of Environmental Contamination and Toxicology, 60, 233-240. https://doi.org/10.1007/s00244-010-9543-0

Han, S.H., Kim, D.H., Shin, S.J. (2013). Bioaccumulation and Physiological Response of Five Willows to Toxic Levels of Cadmium and Zinc. Soil and Sediment Contamination, 22(3), 241-255. https://doi.org/10.1080/15320383.2013.726290

Hu, Y., Liu, X., Bai, J., Shih, K., Zeng, E.Y., Cheng, H. (2013). Assessing heavy metal pollution in the surface soils of a region that had undergone three decades of intense industrialization and urbanization. Environmental Science and Pollution Research, 20(9), 6150-6159. https://doi.org/10.1007/s11356-0131668-z

ISRBC (2009). Sava River Basin report. http://www.savacommission.org/dms/docs/dokumenti/documents_publications/publications/other_publi cations/sava_river_basin_analysis_report_high_res.pdf. Accessed 19 April 2019.

ISRBC (2016). Towards Practical Guidance for Sustainable Sediment Management using the Sava River Basin as a Showcase. Estimation of Sediment Balance for the Sava River. http://www.savacommission.org/dms/docs/dokumenti/documents_publications/publications/other_publi cations/balses_final.pdf. Accessed 19 April 2019.

Jakovljević, T., Bubalo, M.C., Orlović, S., Sedak, M., Bilandžić, N., Brozinčević, I., Redovniković, I.R. (2014). Adaptive response of poplar (Populus nigra L.) after prolonged Cd exposure period. Environmental Science and Pollution Research, 21(5), 3792-3802. https://doi.org/10.1007/s11356-013-2292-7

Jovanović, B. (1970). Order Juglandales. In: M. Josifović (Ed) The Flora of Serbia, Book II, Serbian Academy of Science and Art, Belgrade (in Serbian).

Kabata-Pendias, A. (2011). Trace Elements in Soils and Plants. fourth ed. CRC Press, Boca Raton. ISBN 978-14200-9368-1

Kabata-Pendias, A., Mukherjee, A.B. (2007). Trace elements from soil to human. New York: Springer-Verlag. River: Prentice Hall. ISBN 978-3-540-32714-1

Karadžić, B., Jarić, S., Pavlović, P., Mitrović, M. (2015). Aquatic and wetland vegetation along the Sava River. In: R. Milačič, J. Ščančar, M. Paunović (Vol Eds), The Sava River (pp. 249-317). Springer, Dodrecht, Heidelberg, New York, London ISBN 978-3-662-44034-6.

Kowalska, J.B., Mazurek, R., Gąsiorek, M., Zaleski, T. (2018). Pollution indices as useful tools for the comprehensive evaluation of the degree of soil contamination-A review. Environmental Geochemistry and Health, 40(6), 2395-2420. https://doi.org/10.1007/s10653-018-0106-z 
Kuzovkina, Y.A., Knee, M., Quigley, M.F. (2004). Cadmium and copper uptake and translocation in five willow (Salix L.) species. International Journal of Phytoremediation, 6(3), 269-287. https://doi.org/10.1080/16226510490496726

Laureysens, I., De Temmerman, L., Hastir, T., Van Gysel, M., Ceulemans, R. (2005). Clonal variation in heavy metal accumulation and biomass production in a poplar coppice culture. II. Vertical distribution and phytoextraction potential. Environmental Pollution, 133(3), 541-551. https://doi.org/10.1016/j.envpol.2004.06.013

Li, M.S., Luo, Y.P., Su, Z.Y. (2007). Heavy metal concentrations in soils and plant accumulation

in a restored manganese mine land in Guangxi, South China. Environmental Pollution, 147, 168-175. https://doi.org/10.1016/j.envpol.2006.08.006

Madejón, P., Ciadamidaro, L., Marañón, T., Murillo, J.M. (2013). Long-term biomonitoring of soil contamination using poplar trees: accumulation of trace elements in leaves and fruits. International Journal of Phytoremediation 15(6), 602-14. https://doi.org/10.1080/15226514.2012.723062

Malik, R.N., Husain, S.Z., Nazir, I. (2010). Heavy Metal Contamination and Accumulation in

Soil and Wild Plant Species from Industrial Area of Islamabad, Pakistan. Pakistan Journal of Botany, 42(1), 291-301. ISSN:0556-3321

Marković, M., Zuliani, T., Belanović-Simić, S., Mataruga, Z., Kostić, O., Jarić, S., Vidmar, J., Milačič, R., Ščančar, J., Mitrović, M., Pavlović, P. (2018). Potentially toxic elements in the riparian soils of the Sava River. Journal of Soils and Sediments, 18(12), 3404-3414. https://doi.org/10.1007/s11368-018-2071-7

Marmiroli, M., Antonioli, G., Maestri, E., Marmiroli, N. (2005). Evidence of the involvement of plant lignocellulosic structure in the sequestration of $\mathrm{Pb}$ : An X-ray spectroscopy-based analysis. Environmental Pollution, 134(2), 217-227. https://doi.org/10.1016/j.envpol.2004.08.004

Marmiroli, N., Maestri, E., Antonioli, G., Conte, C., Monciardini, P., Marmiroli, M., Mucchino, C. (1999). Application of synchrotron radiation X-ray fluorescence (m-SRXF) and X-ray microanalysis (SEM/EDX) for the quantitative and qualitative evaluation of trace element accumulation in woody plants. International Journal of Phytoremediation, 1(2), 168-187. https://doi.org/10.1080/15226519908500014

Marschner, H. (1995). Mineral nutrition of higher plants. Second edition. Academic Press Limited, London, Great Britain. ISBN 0-12-473542-8

Meers, E., Vandecasteele, B., Ruttens, A., Vangronsveld, J., Tack, F.M.G. (2007). Potential of five willow species (Salix spp.) for phytoextraction of heavy metals. Environmental and Experimental Botany, 60(1), 57-68. https://doi.org/10.1016/j.envexpbot.2006.06.008

Meers, E., Vervaeke, P., Tack, F.M.G., Lust, N., Verloo, M., Lesage, E. (2003). Field trial experiment: phytoremediation with Salix sp. on a dredged sediment disposal site in Flanders, Belgium. Remediation Journal, 13, 87-97. https://doi.org/10.1002/rem.10077

Mertens, J., Vervaeke, P., De Schrijver, A., Luyssaert, S. (2004). Metal uptake by young trees from dredged brackish sediment: Limitations and possibilities for phytoextraction and phytostabilisation. Science of the Total Environment, 326(1-3), 209-215. https://doi.org/10.1016/j.scitotenv.2003.12.010

Milačič, R., Zuliani, T., Vidmar, J., Oprčkal, P., Ščančar, J. (2017). Potentially toxic elements in water and sediments of the Sava River under extreme flow events. Science of the Total Environment, 605/606, 894-905. https://doi.org/10.1016/j.scitotenv.2017.06.260

Mil-Homens, M., Stevens, R.L., Abrantes, F., Cato, I. (2006). Heavy metal assessment for surface sediments from three areas of the Portuguese continental shelf. Continental Shelf Research, 26, 1184-1205. https://doi.org/10.1016/j.csr.2006.04.002

Minkina, T.M., Nevidomskaya, D.G., Pol'shina, T.N., Fedorov, Y.A., Mandzhieva, S.S., Chaplygin, V.A., Bauer, T.V., Burachevskaya, M.V. (2017). Heavy metals in the soil-plant system of the Don River estuarine region and the Taganrog Bay coast. Journal of Soils and Sediments, 17(5), 1474-1491. https://doi.org/10.1007/s11368-016-1381-x

Naiman, R.J., Decamps, H. (1997). The ecology of interfaces: riparian zones. Annual Review of Ecology, Evolution, and Systematics, 28, 621-658. https://doi.org/10.1146/annurev.ecolsys.28.1.621

Nečemer, M., Kump, P., Ščančar, J., Jaćimović, R., Simčič, J., Pelicon, P., Budnar, M., Jeran, Z., Pongrac, P., Regvar, M., Vogel-Mikuš, K. (2008). Application of X-ray fluorescence analytical techniques in phytoremediation and plant biology studies. Spectrochimica Acta Part B, 63(11), 1240-1247. https://doi.org/10.1016/j.sab.2008.07.006

Oelbermann, M., Raimbault, B.A. (2015). Riparian land-use and rehabilitation: impact on organic matter input and soil respiration. Environmental Management, 55, 496-507. https://doi.org/10.1007/s00267-0140410-z

Ogrinc, N., Kanduč, T., Kocman, D. (2015). Integrated Approach to the Evaluation of Chemical Dynamics and Anthropogenic Pollution Sources in the Sava River Basin In: R. Milačič, J. Ščančar, M. Paunović (Vol 
Eds), The Sava River (pp. 75-95). Springer, Dodrecht, Heidelberg, New York, London ISBN 978-3662-44034-6.

Özcan, M. (2008). Determination of the mineral compositions of some selected oil-bearing seeds and kernels using Inductively Coupled Plasma Atomic Emission Spectrometry (ICP-AES). Grasas y Aceites 57(2), 211-218. https://doi.org/10.3989/gya.2006.v57.i2.39

Ozen, S.A., Yaman, M. (2016). Characterization of the absorption of histidine and lead by Juglan regia L., Platanus L., and Pinus nigra L. using high-performance liquid chromatography-mass spectrometry and inductively coupled plasma-mass spectrometry. Instrumentation Science \& Technology, 44(3), 324332. https://doi.org/10.1080/10739149.2015.1098658

Pavlović, P., Marković, M., Kostić, O., Sakan, S., Đorđević, D., Perović, V., Pavlović, D., Pavlović, M., Čakmak, D., Jarić, S., Paunović, M., Mitrović, M. (2019). Evaluation of potentially toxic element contamination in the riparian zone of the River Sava. Catena, 174, 399-412. https://doi.org/10.1016/j.catena.2018.11.034

Pavlović, P., Mitrović, M., Đordević, D., Sakan, S., Slobodnik, J., Liška, I., Csanyi, B., Jarić, S., Kostić, O., Pavlović, D., Marinković, N., Tubić, B., Paunović, M. (2016). Assessment of the contamination of riparian soil and vegetation by trace metals - A Danube River case study. Science of the Total Environment, 540, 396-409. https://doi.org/10.1016/j.scitotenv.2015.06.125

Pennington, D.N., Hansel, J.R., Gorchov, D.L. (2010). Urbanization and riparian forest woody communities: Diversity, composition, and structure within a metropolitan landscape. Biological Conservation, 143(1), 182-194. https://doi.org/10.1016/j.biocon.2009.10.002

Piczak, K., Lesnievicz, A., Zyrnicki, A. (2003). Metal concentrations in deciduous tree leaves from urban areas in Poland. Environmental Monitoring and Assessment, 86, 273-287. https://doi.org/10.1023/A:1024076504099

Pugh, R.E., Dick, D.G., Fredeen, A.L. (2002). Heavy metal (Pb, Zn, Cd, Fe and Cu) contents of plant foliage near the Anvil Range Lead/Zinc mine, Faro, Yukon Territory. Ecotoxicology and Environmental Safety, 52, 273-279. https://doi.org/10.1006/eesa.2002.2201

Punshon, T., Dickinson, N.M. (1997). Acclimation of Salix to metal stress. New Phytologist, 137, $303-314$. https://doi.org/10.1046/j.1469-8137.1997.00802.x

Punshon, T., Gaines, K.F., Bertsch, P.M., Burger, J. (2003). Bioavailability of uranium and nickel to vegetation in a contaminated riparian ecosystem. Environmental Toxicology and Chemistry, 22, 1146-1154. https://doi.org/10.1002/etc.5620220525

Robinson, B.H., Fernandez, J.E., Madejon, P., Matarien, T., Murillo, J.M., Green, S., Clothier, B. (2003). Phytoextraction: An assessment of biochemical and economic viability. Plant and Soil, 249(1), 117125. https://doi.org/10.1023/A:1022586524971

Sabater, S., Elosegi, A. (2013) River Conservation: Challenges and Opportunities. Fundación BBVA, Bilbao. ISBN: 978-84-92937-47-9

Saqib, A.N.S., Waseem, A., Khan, A.F., Mahmood, Q., Khan, A., Habib, A., Khan, A.R. (2013). Arsenic bioremediation by low cost materials derived from Blue Pine (Pinus wallichiana) and Walnut (Juglans regia). Ecological Engineering, 51, 88-94. https://doi.org/10.1016/j.ecoleng.2012.12.063

Sawidis, T., Breuste, J., Mitrović, M., Pavlović, P., Tsigaridas, K. (2011). Trees as bioindicator of heavy metal pollution in three European cities. Environmental Pollution, 159(12), 3560-3570. https://doi.org/10.1016/j.envpol.2011.08.008

Sawidis, T., Chettri, M.K., Stratis, J., Papaioannou, A., Zachariadis, G. (2002). A Study of Metal Distribution from Lignite Fuels Using Trees as Biological Monitors. Ecotoxicology and Environmental Safety, 48(1), 27-35. https://doi.org/10.1006/eesa.2000.2001

Schulz-Zunkel, C., Krueger, F., Rupp, H., Meissner, R., Gruber, B., Gerisch, M., Bork, H.R. (2013). Spatial and seasonal distribution of trace metals in floodplain soils. A case study with the middle Elbe River, Germany. Geoderma, 211-212, 128-137. https://doi.org/10.1016/j.geoderma.2013.07.010

Schwarz, U. (2016). Sava White Book. The River Sava: Threats and Restoration Potential. Radolfzell/Wien: EuroNatur/Riverwatch

Shu, W.S., Ye, Z.H., Lan, C.Y., Zhang, Z.Q., Wong, M.H. (2002). Lead, zinc and copper accumulation and tolerance in populations of Paspalum distichum and Cynodon dactylon. Environmental Pollution, 120, 445-453. https://doi.org/10.1016/S0269-7491(02)00110-0

Simić, V., Petrović, A., Erg, B., Dimović, D., Makovinska, J., Karadžić, B., Paunović, M. (2015). Indicative Status Assessment, Biodiversity Conservation and Protected Areas within the Sava River Basin. In: R. Milačič, J. Ščančar, M. Paunović (Vol Eds), The Sava River (pp. 453-500). Springer, Dodrecht, Heidelberg, New York, London ISBN 978-3-662-44034-6 
Stobrawa, K., Lorenc-Plucińska, G. (2007). Changes in antioxidant enzyme activity in the fine roots of black poplar (Populus nigra L.) and cottonwood (Populus deltoides Bartr. ex Marsch) in a heavy-metalpolluted environment. Plant and Soil, 298(1-2), 57-68. https://doi.org/10.1007/s11104-007-9336-z

Sunil, C., Somashekar, R. K., Nagaraja, B. C. (2010). Riparian vegetation assessment of Cauvery River Basin of South India. Environmental Monitoring and Assessment, 170(1-4), 545-553. https://doi.org/10.1007/s10661-009-1256-3

Sutherland, R.A. (2000). Bed sediment-associated trace metals in an urban stream, Oahu, Hawaii. Environmental Geology, 39, 611-627. https://doi.org/10.1007/s002540050473

Swarzenski, P.W., Baskaran, M., Rosenbauer, P.J., Orem, W.H. (2006). Historical trace element distribution in sediments from the Mississippi River delta. Estuaries and Coasts, 29(6), 1094-1107. https://doi.org/10.1007/BF02781812

Ščančar, J., Heath, E., Zuliani, T., Horvat, M., Kotnik, J., Perko, P., Milačič, R. (2015). Elements and Persistent Organic Pollutants in the Sediments of the Sava River. In: R. Milačič, J. Ščančar, M. Paunović (Vol Eds), The Sava River (pp. 95-123). Springer, Dodrecht, Heidelberg, New York, London ISBN 978-3662-44034-6

Tang, T., Miller, D.M. (1991). Growth and tissue composition of rice grown in soil treated with inorganic copper, nickel, and arsenic. Communications in Soil Science and Plant Analysis, 22(19-20), 2037-2045. https://doi.org/10.1080/00103629109368556

Tošić, S.B., Mitić, S.S., Velimirović, D.S., Stojanović, G.S., Pavlović, A.N., Pecev-Marinković, E.T. (2014). Elemental composition of edible nuts: fast optimization and validation procedure of an ICP-OES method. Journal of the Science of Food and Agriculture, 95(11), 2271-2278. https://doi.org/10.1002/jsfa.6946

Vandecasteele, B., De Vos, B., Tack, F.M.G. (2002). Cadmium and Zinc uptake by volunteer willow species and elder rooting in polluted dredged sediment disposal sites. Science of the Total Environment, 299(1-3), 191-205. https://doi.org/10.1016/S0048-9697(02)00275-9

Vandecasteele, B., Meers, E., Vervaeke, P., De Vos, B., Quataert, P., Tack, F.M.G. (2005). Growth and trace metal accumulation of two Salix clones on sediment-derived soils with increasing contamination levels. Chemosphere, 58(8), 995-1002. https://doi.org/10.1016/j.chemosphere.2004.09.062

Vysloužilová, M., Tlustoš, P., Száková, J., Pavlíková, D. (2003). As, Cd, Pb and Zn uptake by Salix spp. clones grown in soils enriched by high loads of these elements. Plant, Soil and Environment, 49(5), 191-196. http://dx.doi.org/10.17221/4112-PSE

Wu, F., Yang, W., Zhang, J., Zhou, L. (2010). Cadmium accumulation and growth responses of a poplar (Populus deltoides x Populus nigra) in cadmium contaminated purple soil and alluvial soil. Journal of Hazardous Materials, 177, 268-273. http://dx.doi.org/10.1016/j.jhazmat.2009.12.028

Yoon, J., Cao, X., Zhou, Q., Ma, L.Q. (2006). Accumulation of Pb, Cu, and Zn in native plants growing on a contaminated Florida site. Science of the Total Environment, 368(2-3), 456-464. https://doi.org/10.1016/j.scitotenv.2006.01.016

Zacchini, M., Iori, V., Mugnozza, G.S., Pietrini, F., Massacci, A. (2011). Cadmium accumulation and tolerance in Populus nigra and Salix alba. Biologia Plantarum, 55(2), 383-386. https://doi.org/10.1007/s10535011-0060-4

Zayed, A., Gowthaman, S., Terry, N. (1998). Phytoaccumulation of trace elements by wetland plants: I. Duckweed. Journal of Environmental Quality, 27, 715-721. https://doi.org/10.2134/jeq1998.00472425002700030032x

Zimmer, D., Baum, C., Meissner, R., Leinweber, P. (2012). Soil-ecological evaluation of willows in a floodplain. Journal of Soil Science and Plant Nutrition, 175(2), 245-252. https://doi.org/10.1002/jpln.201100063

Zhang, H., Cui, B., Zhang, K. (2011). Heavy metal distribution of natural and reclaimed tidal riparian wetlands in south estuary, China. Journal of Environmental Sciences 23(12), 1937-1946. https://doi.org/10.1016/S1001-0742(10)60644-4

Zovko, M., Romić, M. (2011). Soil contamination by trace metals: geochemical behavior as an element of risk assessment. In: I.A., Dar (Ed), Earth and Environmental Sciences (pp 437-456). InTech, Rijeka https://doi.org/10.5772/25448 